\title{
Hypertrophic cardiomyopathy mutations in the pliant and light chain- binding regions of the lever arm of human $\beta$-cardiac myosin have divergent effects on myosin function
}

\author{
Makenna M. Morck ${ }^{1,2}$, Debanjan Bhowmik ${ }^{1,2, a}$, Aminah Dawood $^{1,2}$, James A. Spudich ${ }^{1,2}$, \\ Kathleen M. Ruppel ${ }^{1,2,3}$
}

Affiliations:

1. Department of Biochemistry, Stanford University School of Medicine, Stanford CA 94305

2. Stanford Cardiovascular Institute, Stanford University School of Medicine, Stanford CA 94305

3. Department of Pediatrics (Cardiology), Stanford University School of Medicine, Stanford CA 94305

a. Current address: Transdisciplinary Research Program, Rajiv Gandhi Centre for Biotechnology, Thiruvananthapuram, Kerala 695014, India

\begin{abstract}
Mutations in the lever arm of $\beta$-cardiac myosin are a frequent cause of hypertrophic cardiomyopathy (HCM), a disease characterized by hypercontractility and eventual hypertrophy of the left ventricle. Here, we studied five such mutations: three in the pliant region of the lever arm (D778V, L781P, and S782N) and two in the light chain-binding region (A797T and F834L). We investigated their effects on both motor function and myosin S2 tail-based autoinhibition. The pliant region mutations had varying effects on the motor function of a myosin construct lacking the S2 tail: overall, D778V increased power output, L781P reduced power output, and S782N had little effect on power output, while all three reduced the external force sensitivity of the actin detachment rate. With a myosin containing the motor domain and the proximal S2 tail, the pliant region mutations also attenuated autoinhibition in the presence of filamentous actin but had no impact in the absence of actin. By contrast, the light chain-binding region mutations had little effect on motor activity but produced marked reductions in autoinhibition in both the presence and absence of actin. Thus, mutations in the lever arm of $\beta$-cardiac myosin have divergent allosteric effects on myosin function, depending on whether they are in the pliant or light chain-binding regions.
\end{abstract}




\section{INTRODUCTION}

The myosin lever arm was first recognized almost 30 years ago as the first structures of myosin S1 were described ${ }^{1,2}$, and the lever arm's function in amplifying the motion of the converter domain was subsequently confirmed ${ }^{3,4}$. Much work since has focused on understanding the functional significance of different lever arm features across myosin classes ${ }^{5}$. However, less attention has been paid to the functional consequences of point mutations in the myosin lever arm. Such mutations in the human $\beta$-cardiac myosin lever arm are a frequent cause of hypertrophic cardiomyopathy $(\mathrm{HCM})$, a disease characterized by hypercontractility and eventual hypertrophy of the left ventricle 6 . While many HCM-causing mutations in the myosin motor domain have been described and characterized ${ }^{7-15}$, HCM-causing mutations in the lever arm remain understudied, despite the fact that the lever arm has a relatively high rate of these mutations and plays a key role in transducing the chemical energy of ATP hydrolysis into physical motion ${ }^{3,4}$.

$\mathrm{HCM}$ is a leading cause of genetic heart disease, affecting up to 1 in 200 in the US population ${ }^{16}$. Mutations leading to HCM have been found in genes encoding a variety of sarcomeric proteins; however, the vast majority occur in either the $\beta$-cardiac myosin heavy chain or cardiac myosin binding protein- ${ }^{17}$. Because left ventricular hypercontractility typically precedes hypertrophy in $\mathrm{HCM}_{\text {patients }}{ }^{6}$, it has been hypothesized that sarcomeric mutations lead to hypercontractility at the molecular scale. Early work in this area suggested that mutations might increase myosin's motor activity by increasing its actin-activated ATPase rate, motility velocity, or force production $^{18,19}$. While some mutations may function by this mechanism ${ }^{8,11,15}$, lately, a more compelling hypothesis has gained traction: many HCM-causing mutations appear to reduce myosin's ability to form an autoinhibited state ${ }^{10-14,20-24}$. Loss of an autoinhibited state could lead to additional myosin heads acting to generate force during contraction, thus leading to hypercontractility.

An autoinhibited state of myosin was first described in smooth muscle myosin over 20 years ago ${ }^{25}$, but its relevance to $\beta$-cardiac myosin and $\mathrm{HCM}$ was only more recently recognized. In the structural view of this smooth muscle autoinhibited state, the myosin heads fold back onto their own S2 tail in a conformation known as the interacting heads motif $(\mathrm{IHM})^{26,27}$. One of the two heads in the dimer has its actin-binding interface buried in the folded structure; this head is referred to as the "blocked head," while the other head is called the "free head," since its actin binding interface is not hidden structurally. Many myosin types have been shown to assume this 
folded back IHM structure ${ }^{27-29}$, and it is now thought to be a common feature across the myosin II class ${ }^{30}$.

The IHM structure has been correlated to an ultra-low basal ATPase rate (three orders of magnitude below the actin-activated ATPase rate) in the absence of actin called the "super relaxed state" $(S R X)^{24,29,31-33}$. Heads lacking the $S 2$ tail mostly have a faster basal ATPase rate (two orders of magnitude below the actin-activated ATPase rate) referred to as the "disordered relaxed state" (DRX). Additionally, actin-activated ATPases comparing myosin with and without its S2 tail have shown that when the S2 tail is present, the apparent ATPase rate decreases, suggesting that the $\mathrm{S} 2$ tail promotes autoinhibition ${ }^{10-13,34}$. Therefore, myosin containing the S2 tail is thought to be in equilibrium between an open state available for actin binding and the closed IHM conformation. While these structural states are correlated to specific basal and actinactivated ATPase rates, there may be circumstances in which the structural states are uncoupled from their respective rates ${ }^{35,36}$. Thus, while these functional assays measure autoinhibition by the myosin S2 tail, they are only correlative measures for structural states (i.e., the IHM).

HCM-causing mutations in the myosin lever arm could lead to hypercontractility by 1) disrupting S2 tail-based autoinhibition 2) increasing intrinsic motor activity without affecting autoinhibition, or 3) affecting both intrinsic motor activity and autoinhibition. The lever arm's role in the formation of the autoinhibited state has previously been explored in mollusk myosin, which is primarily regulated via molecular switching from the autoinhibited off state to an open state in the presence of $\mathrm{Ca}^{2+}$. A number of structural studies collectively concluded that three joints in the molluscan myosin lever arm appear to be potential sources of flexibility which may be necessary for the formation of the IHM: a region at the extreme N-terminus of the lever arm, termed the "pliant" region, a typically bent region between the essential light chain (ELC) and the regulatory light chain (RLC) binding regions, and a region at the C-terminus of the lever arm in the RLC binding area termed the "hook" or "ankle" joint ${ }^{37-41}$. Recently, high-resolution cryo-EM structures of the folded-back IHM state in smooth muscle myosin demonstrated that the lever arm must take on a different conformation in each of the asymmetric folded heads in the IHM, further confirming the importance of lever arm positioning in the folded state ${ }^{42,43}$. Mutations in the lever arm could reduce myosin's S2 tail-based autoinhibition by negatively impacting any of these structural requirements for forming the IHM. Alternately, lever arm mutations could increase intrinsic motor activity, leading to hypercontractility. For example, mutations could increase the rigidity of the lever arm and/or alter its light chain-binding properties, which could in turn influence power output. Indeed, 
several previous studies investigating RLC mutations suggested that mutations can modulate lever arm compliance ${ }^{44-47}$. Lever arm mutations could also allosterically affect the motor domain, potentially leading to increased motor activity.

In the present study, we examined five adult-onset HCM-causing mutations in the myosin lever arm to determine how they affect myosin function: D778V, L781P, S782N, A797T, and F834L (Fig. 1). We hypothesized that mutations in the lever arm could affect myosin either by 1) altering its intrinsic motor activity, and/or 2) reducing its ability to form the autoinhibited state. We found that three mutations in the pliant region of the lever arm, D778V, L781P, and S782N, lead to changes in both myosin's motor activity and formation of the auto-inhibited state. Two mutations in the light chain-binding regions, A797T and F834L, clearly impact myosin's ability to form the auto-inhibited state, likely explaining their contributions to hypercontractility. Thus, mutations in the lever arm have varying impacts on myosin function, depending on whether they are in the pliant region or the light chain binding regions. 


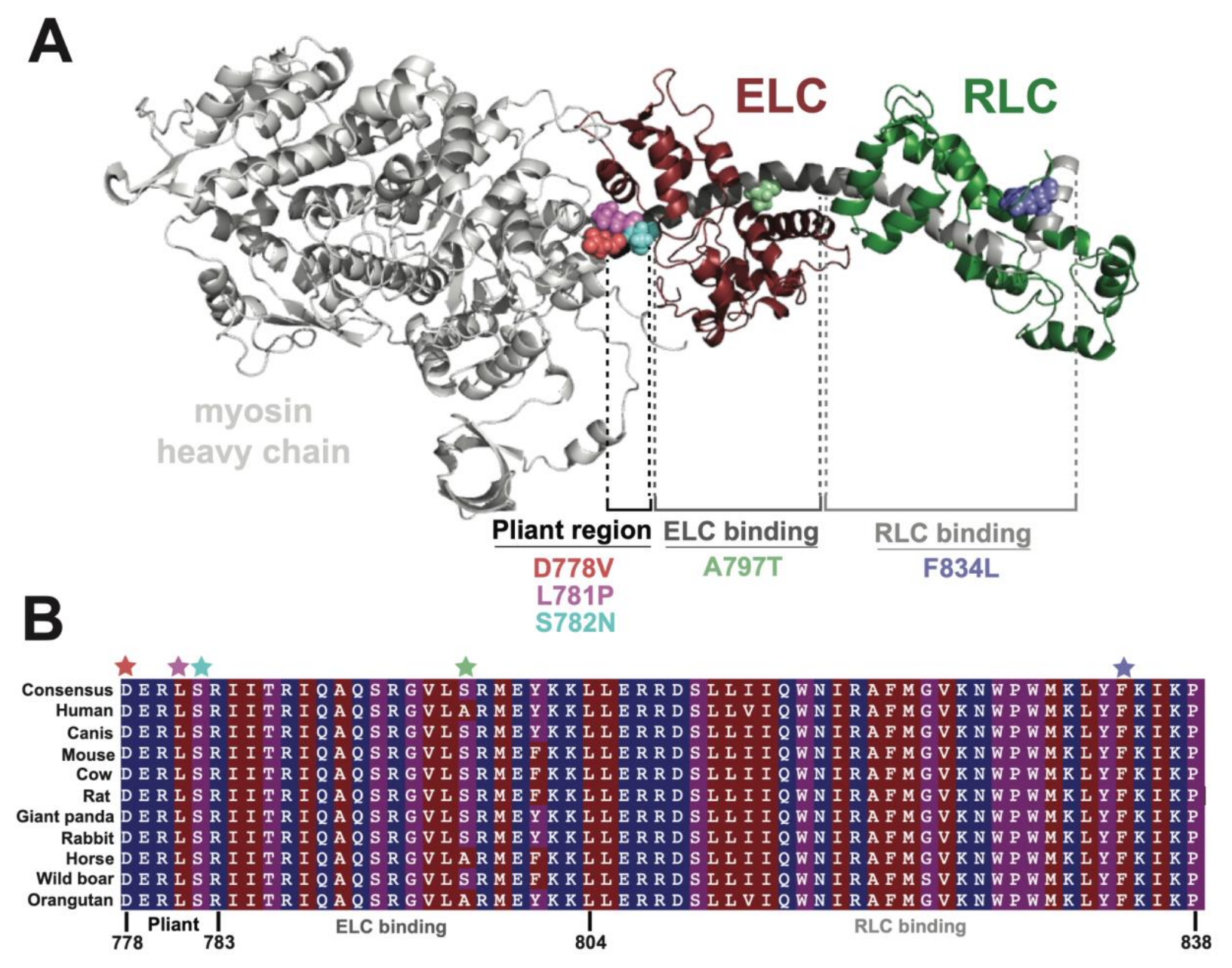

Figure 1: Location of HCM-causing mutations along the lever arm. A) Homology model of the prestroke $\beta$-cardiac myosin S148 highlighting the 5 mutations examined in the present study: D778V, L781P, S782N, A797T, and F834L and their locations along the lever arm. The essential light chain (ELC) and regulatory light chain (RLC) are shown in maroon and green, respectively. B) Alignment of $\beta$-cardiac myosin lever arms for several model organisms, demonstrating the high degree of conservation of the lever arm across species. Residues are colored by hydrophobicity, where red is most hydrophobic, and blue is most hydrophilic. Mutated residues studied herein are indicated with colored stars. 


\section{RESULTS}

\section{Mutations do not significantly impact light chain loading}

We first sought to determine whether any of the five mutations impacted the expected 1:1:1 stoichiometry of myosin heavy chain:ELC:RLC of our purified proteins. To test this, we used a Coomassie SDS-PAGE gel-based assay that quantified the ratio of myosin heavy chain to ELC and RLC (Fig. S1A). For this assay, we used a two-headed myosin construct that contains the myosin head, full lever arm, and the first two heptads of the S2 tail, followed by a GCN4 leucine zipper moiety to ensure dimerization, a GFP tag, and a C-terminal affinity clamp peptide tag (2hep $\operatorname{myosin}{ }^{10}$ ). When compared to the paired WT 2-hep control, none of the mutants had significantly changed ELC loading ( $p$ vs. WT for $D 778 \mathrm{~V}=0.085, \mathrm{~L} 781 \mathrm{P}=0.17, \mathrm{~S} 782 \mathrm{~N}=0.53$, $\mathrm{A} 797 \mathrm{~T}=0.78, \mathrm{~F} 834 \mathrm{~L}=0.95$ ) or RLC loading ( $p$ vs. WT for D778V $=0.14, \mathrm{~L} 781 \mathrm{P}=0.28, \mathrm{~S} 782 \mathrm{~N}$ $=0.32, \mathrm{~A} 797 \mathrm{~T}=0.82, \mathrm{~F} 834 \mathrm{~L}=0.78)$, suggesting that at least for these five mutations in the context of our purified system, light chain loading is not significantly impacted (Fig. S1C).

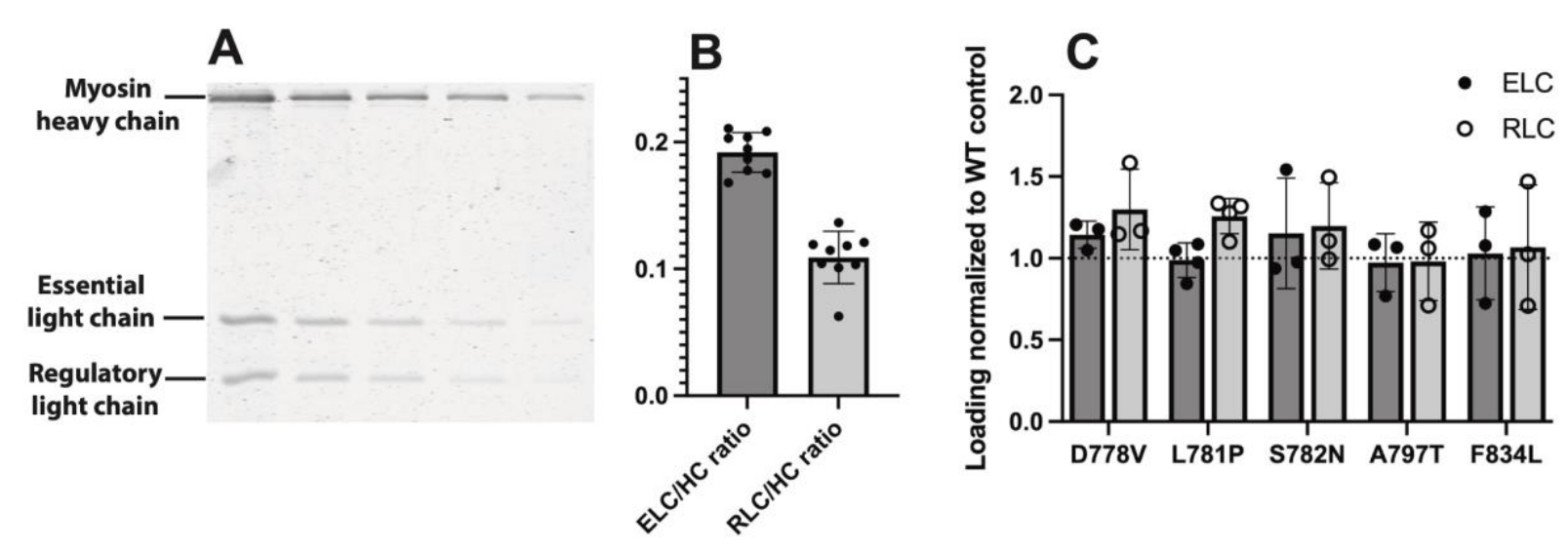

Figure S1: Light chain loading of WT vs. mutant myosins. A) Representative gel used to quantitate light chain loading. For each sample, myosin was titrated in a denaturing SDS-PAGE gel, stained with Coomassie, and scanned for $700 \mathrm{~nm}$ fluorescence. See full methods. B) Light chain loading across all WT 2-hep samples measured. Mean $\mathrm{ELC} / \mathrm{HC}=0.19 \pm 0.02, \mathrm{RLC} / \mathrm{HC}=0.11 \pm 0.02$ (mean $\pm \mathrm{SD}$ ). Expected $\mathrm{ELC} / \mathrm{HC}$ and $\mathrm{RLC} / \mathrm{HC}$ ratios if Coomassie staining were unbiased are 0.168 and 0.146 . C) Light chain loading of mutant 2-hep myosins normalized to WT controls. For each mutant, light chain loading was not statistically different from WT. 


\section{Only D778V impacts the actin-activated ATPase rate of two-headed myosin with a short tail}

We then examined the effects of each of the 5 mutations on myosin's actin-activated ATPase activity of 2 -hep $\beta$-cardiac myosin. We have previously shown that 2 -hep myosin produces similar ATPase rates to $\mathrm{S} 1$ constructs, albeit with a slightly tighter apparent affinity for actin $\left(\mathrm{K}_{\mathrm{M}}\right)^{11}$. Of the 5 mutations, only D778V produced a significantly different actin-activated ATPase rate as compared to WT, increasing the turnover number $k_{\text {cat }}$ by $16 \pm 9 \%$ ( $p=0.041$, average of three technical replicates of each of two biological replicates, compared to WT controls; Fig. 2A; see raw values in table S1). In contrast, L781P, S782N, A797T, and F834L all had actin-activated ATPase curves that very closely resembled their matched WT controls (Fig. 2B-E; see raw values in table S1).
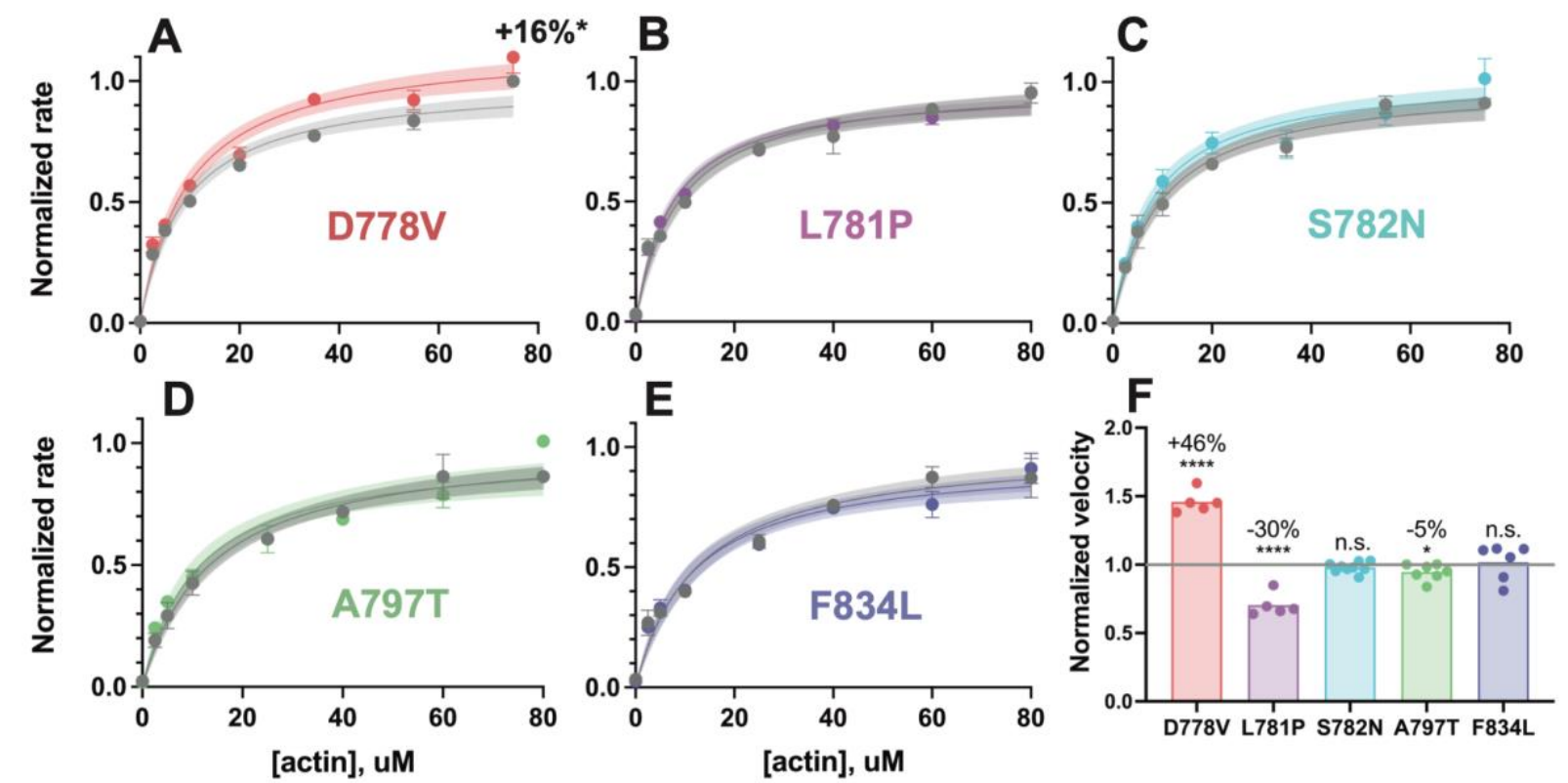

Figure 2: Actin-activated ATPase activity and in vitro motility velocity of 2-hep human $\beta$-cardiac myosin lever arm mutants versus WT. A-E) Representative actin-activated ATPase curves of 2-hep constructs for each lever arm mutant, normalized to their WT controls. Each plot shows a single biological replicate (one of two, see table S1), where error bars represent the standard deviation of three technical replicates. Where error bars are not shown, error is smaller than the size of the data point. Mutant data are plotted against their prep-matched WT 2-hep control (gray) and normalized to the WT $k_{\text {cat }}$ value. Curves are fit to Michaelis-Menten kinetics, and the shaded areas represent $95 \%$ confidence intervals of the fits. Only D778V produced a significant difference: the $k_{\text {cat }}$ was increased by $16 \pm 9 \%$ (average of two independent biological replicates compared to WT controls). F) In vitro motility velocities of lever arm mutants. Each data point represents the average velocity of the mutant 2-hep on a single slide normalized to the WT 2-hep velocity from the same slide. Bars represent the average of the data points. D778V 
increased the velocity by $46 \pm 8 \%$ over WT, L781P reduced the velocity by $30 \pm 8 \%$ compared to WT, A797T reduced the velocity by $5 \pm 6 \%$, and S782N and F834L had no significant effects on motility velocity (mean \pm SD). ${ }^{* * *}$ indicates $p \leq 0.0001,{ }^{*}$ indicates $p \leq 0.05$

\begin{tabular}{|c|c|c|c|c|c|c|c|c|}
\hline Mutation & Replicate & Protein & $k_{\text {cat }}, \mathbf{s}^{-1}$ & $\mathbf{K}_{\mathrm{M}}, \mathbf{u M}$ & $\begin{array}{c}\text { Mutant 2-hep/ } \\
\text { WT } \\
\text { 2-hep } \boldsymbol{k}_{\text {cat }} \\
\text { ratio }\end{array}$ & $\begin{array}{c}\text { Mutant 25- } \\
\text { hep/mutant 2- } \\
\text { hep } \\
k_{\text {cat ratio }}\end{array}$ & $\begin{array}{c}\text { Average } \\
\text { mutant } \\
\text { 2-hep/WT 2- } \\
\text { hep } \\
\boldsymbol{k}_{\text {cat ratio }}\end{array}$ & $\begin{array}{c}\text { Average } \\
\text { mutant 25- } \\
\text { hep/mutant 2- } \\
\text { hep } k_{\text {cat ratio }}\end{array}$ \\
\hline \multirow{6}{*}{ D778V } & & WT 2-hep & $5.81 \pm 0.36$ & $13.6 \pm 2.7$ & \multirow{3}{*}{$1.17 \pm 0.11$} & \multirow{3}{*}{$0.80 \pm 0.18$} & \multirow{6}{*}{$1.16 \pm 0.09$} & \multirow{6}{*}{$0.81 \pm 0.15$} \\
\hline & 1 & D778V 2-hep & $6.81 \pm 0.33$ & $13.0 \pm 1.9$ & & & & \\
\hline & & D778V 25-hep & $5.45 \pm 0.70$ & $27.3 \pm 8.5$ & & & & \\
\hline & \multirow{3}{*}{2} & WT 2-hep & $5.40 \pm 0.17$ & $8.8 \pm 1.0$ & \multirow{3}{*}{$1.15 \pm 0.07$} & \multirow{3}{*}{$0.81 \pm 0.12$} & & \\
\hline & & D778V 2-hep & $6.21 \pm 0.21$ & $9.7 \pm 1.2$ & & & & \\
\hline & & D778V 25-hep & $5.02 \pm 0.42$ & $33.7 \pm 6.2$ & & & & \\
\hline \multirow{6}{*}{ L781P } & & WT 2-hep & $5.56 \pm 0.30$ & $6.7 \pm 1.8$ & \multirow{3}{*}{$0.98 \pm 0.10$} & \multirow{3}{*}{$0.89 \pm 0.13$} & \multirow{6}{*}{$0.97 \pm 0.08$} & \multirow{6}{*}{$0.85 \pm 0.10$} \\
\hline & 1 & L781P 2-hep & $5.42 \pm 0.26$ & $4.3 \pm 1.3$ & & & & \\
\hline & & L781P 25-hep & $4.83 \pm 0.40$ & $21.8 \pm 5.1$ & & & & \\
\hline & \multirow{3}{*}{2} & WT 2-hep & $4.84 \pm 0.15$ & $8.8 \pm 1.0$ & \multirow{3}{*}{$0.97 \pm 0.05$} & \multirow{3}{*}{$0.81 \pm 0.05$} & & \\
\hline & & L781P 2-hep & $4.71 \pm 0.10$ & $7.2 \pm 0.6$ & & & & \\
\hline & & L781P 25-hep & $3.81 \pm 0.12$ & $15.9 \pm 1.6$ & & & & \\
\hline \multirow{6}{*}{ S782N } & \multirow{3}{*}{1} & WT 2-hep & $3.95 \pm 0.15$ & $3.3 \pm 0.6$ & \multirow{3}{*}{$1.04 \pm 0.07$} & \multirow{3}{*}{$0.72 \pm 0.14$} & \multirow{6}{*}{$1.04 \pm 0.07$} & \multirow{6}{*}{$0.81 \pm 0.12$} \\
\hline & & S782N 2-hep & $4.11 \pm 0.12$ & $2.8 \pm 0.4$ & & & & \\
\hline & & S782N 25-hep & $2.96 \pm 0.32$ & $8.7 \pm 3.1$ & & & & \\
\hline & \multirow{3}{*}{2} & WT 2-hep & $3.78 \pm 0.13$ & $9.4 \pm 1.0$ & \multirow{3}{*}{$1.03 \pm 0.07$} & \multirow{3}{*}{$0.90 \pm 0.11$} & & \\
\hline & & S782N 2-hep & $3.89 \pm 0.14$ & $8.1 \pm 1.0$ & & & & \\
\hline & & S782N 25-hep & $3.52 \pm 0.26$ & $24.6 \pm 4.3$ & & & & \\
\hline \multirow{6}{*}{ A797T } & \multirow{3}{*}{1} & WT 2-hep & $5.70 \pm 0.39$ & $9.7 \pm 2.2$ & \multirow{3}{*}{$0.89 \pm 0.13$} & & & \\
\hline & & A797T 2-hep & $5.10 \pm 0.32$ & $4.9 \pm 1.2$ & & $1.22 \pm 0.20$ & & \\
\hline & & A797T 25-hep & $6.23 \pm 0.84$ & $18.6 \pm 6.8$ & & & & \\
\hline & & WT 2-hep & $5.02 \pm 0.19$ & $13.4 \pm 1.6$ & & & $0.93 \pm 0.11$ & $1.19 \pm 0.16$ \\
\hline & 2 & A797T 2-hep & $4.85 \pm 0.25$ & $10.9 \pm 1.9$ & $0.97 \pm 0.09$ & $1.16 \pm 0.12$ & & \\
\hline & & A797T 25-hep & $5.60 \pm 0.38$ & $27.3 \pm 4.6$ & & & & \\
\hline & & WT 2-hep & $4.65 \pm 0.29$ & $1.5 \pm 0.9$ & & & & \\
\hline & 1 & F834L 2-hep & $5.12 \pm 0.27$ & $1.5 \pm 0.8$ & $1.10 \pm 0.12$ & $0.91 \pm 0.12$ & & \\
\hline & & F834L 25-hep & $4.64 \pm 0.31$ & $3.6 \pm 1.3$ & & & $102+010$ & 10 \\
\hline 34L & & WT 2-hep & $4.64 \pm 0.20$ & $12.4 \pm 1.8$ & & & $1.03 \pm 0.10$ & $0.82 \pm 0.10$ \\
\hline & 2 & F834L 2-hep & $4.43 \pm 0.18$ & $11.4 \pm 1.6$ & $0.96 \pm 0.08$ & $0.73 \pm 0.07$ & & \\
\hline & & F834L 25-hep & $3.22 \pm 0.10$ & $9.0 \pm 1.1$ & & & & \\
\hline & 1 & WT 2-hep & $4.56 \pm 0.22$ & $13.2 \pm 1.0$ & & $0.69 \pm 0.10$ & & \\
\hline WT & 0 & $\begin{array}{l}\text { WT 25-hep } \\
\text { WT 2-hep }\end{array}$ & $\begin{array}{l}3.13 \pm 0.23 \\
6.04 \pm 0.18\end{array}$ & $\begin{array}{c}28.2 \pm 5.1 \\
6.1 \pm 1.6\end{array}$ & N/A & & N/A & $0.63 \pm 0.10$ \\
\hline & 2 & WT 25-hep & $3.48 \pm 0.26$ & $26.4 \pm 5.8$ & & & & \\
\hline
\end{tabular}

Table S1: Full actin-activated ATPase results. For each mutation, two independent biological experiments were performed with freshly prepared myosins, where each biological replicate was measured with technical triplicates (6 total replicates for each protein). The 2- and 25-hep mutant myosins were prepared in tandem with a WT 2-hep control for comparison, given that actin-activated ATPase results show a slight drift from day-to-day. Results in the fourth and fifth columns, respectively, show the fitted values for $k_{\text {cat }}\left(\mathrm{s}^{-1}\right)$ and $\mathrm{K}_{\mathrm{M}}(\mu \mathrm{M}) \pm \mathrm{SE}$ of the fit for each biological replicate. The $k_{\text {cat }}$ ratio of mutant 2-hep/WT 2-hep and mutant 25-hep/mutant 2-hep for each independent biological replicate is shown in the sixth and seventh columns respectively, where the error is propagated from SE of the fit for each measurement. Finally, the average of each $k_{\text {cat }}$ ratio is shown in the two rightmost columns with propagated error. WT measurements are reproduced from $\left[{ }^{13}\right]$. 


\section{Lever arm mutations have varying effects on actin gliding velocity}

We next assessed the effects of lever arm mutations on actin gliding velocity in an in vitro actin gliding motility assay ${ }^{49}$. For these experiments, we again used the 2-hep myosin construct, attached to the surface via interaction of its C-terminal affinity clamp to SNAP-PDZ. In the pliant region, the $\mathrm{D} 778 \mathrm{~V}$ mutation increased actin gliding velocity by $46 \pm 8 \%$ (mean $\pm S D, p<0.0001$ ), the L781P mutation reduced velocity by $30 \pm 8 \%$ (mean $\pm S D, p<0.0001$ ), and the S782N mutation had no significant effect on velocity $(p=0.19)$ as compared to actin gliding velocities of same day WT 2-hep controls (Fig. 2F). In contrast, mutations in the light chain binding domains had little to no effect on gliding velocities, as the A797T mutation reduced velocity by only $5 \pm 6 \%$ (mean $\pm S D, p=0.033$ ) and the F834L mutation had no significant effect on velocity $(p=0.72$; Fig. 2F). Actin gliding velocity is primarily a function of detachment kinetics and step size ${ }^{15}$. Thus, it appears that as a whole, mutations in the pliant region may have more impact on myosin's motor properties (i.e., attachment and detachment kinetics and step size), while the mutations in the light chain-binding regions may impact myosin's activity primarily through a different mechanism. Accordingly, we next sought to further dissect the effects of pliant region mutations using harmonic force spectroscopy.

\section{Harmonic force spectroscopy reveals altered detachment kinetics and step sizes due to pliant region mutations}

We have previously described an optical trap setup called harmonic force spectroscopy (HFS) that allows for the measurement of myosin's detachment rate from actin as a function of external load force ${ }^{15,50,51}$ as well as its step size ${ }^{13}$. Myosin's detachment rate is reduced in the presence of resistive load forces and increased in the presence of assistive load forces, in accordance with the force-velocity relationship of contracting heart muscle ${ }^{50,52}$. Here, we used HFS to measure the load-dependent detachment rates and step size of a short S1 (sS1) myosin construct containing the motor domain of myosin plus the first half of the lever arm (amino acids 1-808), allowing for the binding of the ELC but not the RLC, followed by a GFP tag.

We measured detachment kinetics of several molecules each of WT, D778V, L781P, and S782N and fit their behavior to the Arrhenius equation with a harmonic force correction as described previously ${ }^{15,50,51}$, where $k_{\mathrm{B}}$ is the Boltzmann constant and $T$ is temperature:

$$
k_{\text {det }}(F, \Delta F)=k_{0} I_{0}\left(\frac{\Delta F \delta}{k_{B} T}\right) \exp \left(\frac{-F \delta}{k_{B} T}\right) \quad \text { (Equation 1) }
$$


This equation is a function of $k_{0}$, the detachment rate at zero load force, $\delta$, a measure of the force sensitivity, and $F$, the average external load force applied. Since the molecule experiences sinusoidal force, the equation is corrected with a zero-order Bessel function $I_{0}$ which is a function of $\Delta F$, the amplitude of the sinusoidal force. We then averaged the individual molecules (WT $\mathrm{n}=$ 8 molecules, D778V $\mathrm{n}=12, \mathrm{~L} 781 \mathrm{P} \mathrm{n}=22$, and S782N $\mathrm{n}=11$ ) to obtain the characteristic $k_{0}$ and $\delta$ values of WT and each lever arm mutant myosin. Interestingly, each pliant region mutation resulted in significant changes to the load force vs. detachment rate curve (Fig. 3A), consistent with previous studies showing that mutations frequently impact force-dependent kinetics ${ }^{13,15}$. The fitted values for $k_{0}\left(\mathrm{WT}=146.5 \pm 4.5 \mathrm{~s}^{-1}\right.$ (mean $\left.\pm \mathrm{SEM}\right)$; D778V = $315.6 \pm 14.0 \mathrm{~s}^{-1}, \mathrm{p}<0.0001$; $\mathrm{L} 781 \mathrm{P}=101.7 \pm 5.8 \mathrm{~s}^{-1}, \mathrm{p}<0.0001$; and $\mathrm{S} 782 \mathrm{~N}=145.3 \pm 6.7 \mathrm{~s}^{-1}, \mathrm{p}=0.88$; Fig. $3 \mathrm{~B}$ ) roughly correlate to the changes we observed in motility velocity, consistent with the fact that motility velocity is detachment rate-limited ${ }^{15}$. Interestingly, fitted values for $\delta$ demonstrate that all three pliant region mutations reduce force sensitivity $(\mathrm{WT}=1.08 \pm 0.04 \mathrm{~nm}$ (mean $\pm \mathrm{SEM}$ ); $\mathrm{D} 778 \mathrm{~V}=$ $0.68 \pm 0.05 \mathrm{~nm}, \mathrm{p}<0.0001 ; \mathrm{L} 781 \mathrm{P}=0.81 \pm 0.03 \mathrm{~nm}, \mathrm{p}=0.0002 ; \mathrm{S} 782 \mathrm{~N}=0.83 \pm 0.05 \mathrm{~nm}, \mathrm{p}=$ 0.0010; Fig. 3C). We also measured the step size $d$ of each mutation, and notably only L781P had any measurable impact on step size $(\mathrm{WT}=4.3 \pm 1.2 \mathrm{~nm}$ (mean $\pm \mathrm{SD}$ ); D778V = $4.5 \pm 1.4$ $n m, p=0.72 ; L 781 P=2.5 \pm 1.4 n m, p=0.0026 ; S 782 \mathrm{~N}=4.3 \pm 1.2 \mathrm{~nm}, \mathrm{p}=0.95 ;$ Fig. 3D). 

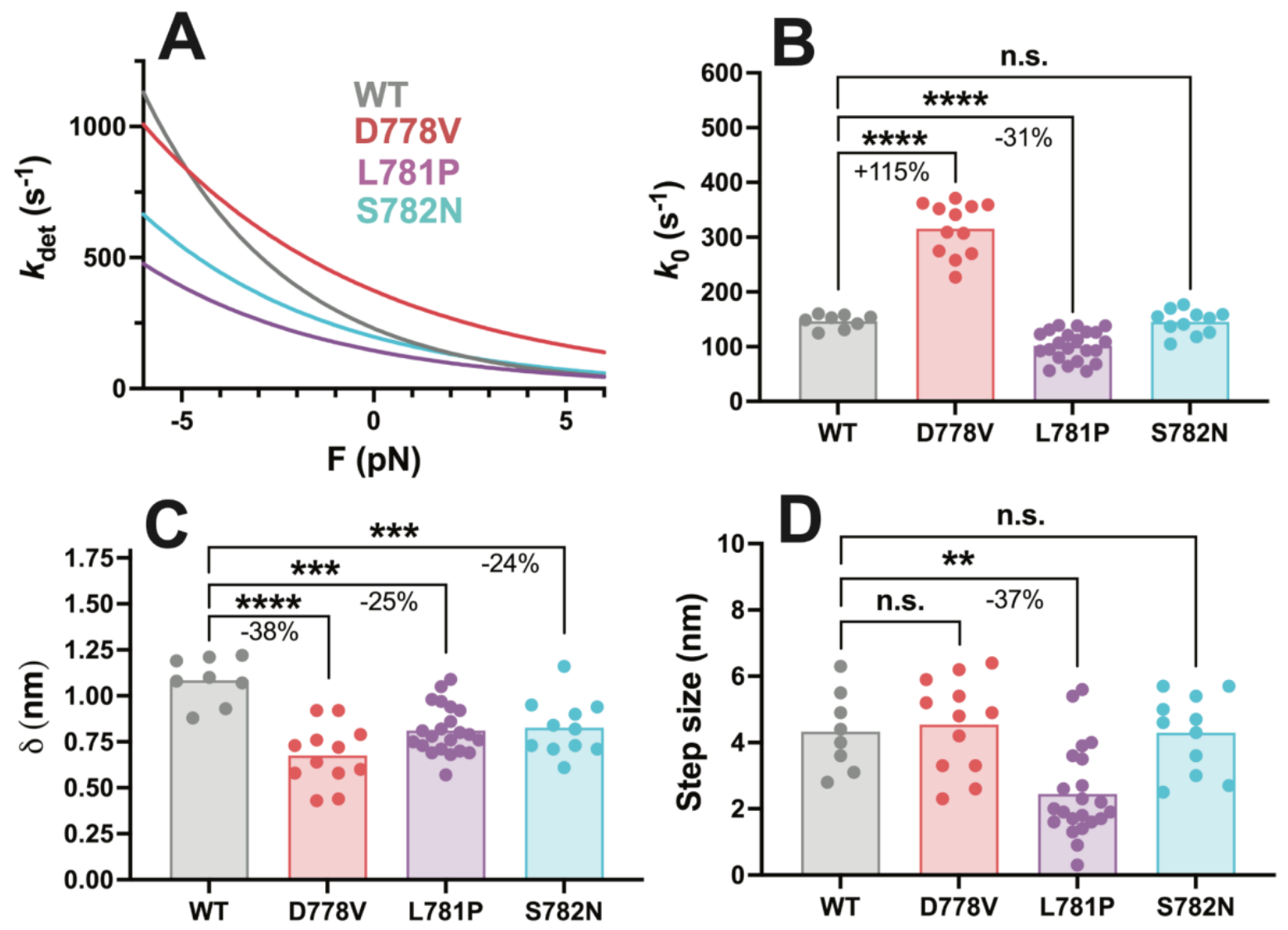

Figure 3: Harmonic force spectroscopy measurements of pliant region mutations in sS1 human $\beta$ cardiac myosin. A) Detachment rate of sS1 myosin as a function of load force, average of all molecules. Each mutation produced a markedly different detachment rate curve compared to the WT. WT curve is shown in gray, D778V curve is shown in red, L781P curve is shown in purple, and S782N curve is shown in cyan. B) $k_{0}$, the detachment rate at zero load force, for WT and each mutant sS1. D778V increased the detachment rate by $115 \pm 10 \%$ (mean \pm SEM), while L781P reduced the detachment rate by $31 \pm 5 \%$, and S782N had no significant effect on detachment rate. C) $\delta$, the measure of force sensitivity, for WT and each mutant SS1. All the pliant region mutations reduced the force sensitivity: D778V by $38 \pm 6 \%$, L781P by 25 $\pm 5 \%$, and S782N by $24 \pm 6 \%$ (mean \pm SEM). D) Step size for WT and each mutation. Only L781P affected step size, reducing it by $37 \pm 12 \%$ (mean \pm SEM). each data point represents the average value for one molecule, and bars show the average of the data points. ${ }^{* *}$ indicates $p \leq 0.01,{ }^{* * *}$ indicates $p \leq 0.001,{ }^{* * *}$ indicates $p \leq 0.0001$ 


\section{Pliant region mutations have varied impacts on duty ratio, ensemble force, and power output}

Given measured values for $k_{\text {cat }}, k_{0}$, step size, and the load-dependent detachment rate $k_{\text {det }}$, duty ratio $r$ as a function of load force $F$ can be calculated as follows ${ }^{15}$ :

$$
r(F)=\frac{k_{\text {attach }}}{k_{\text {attach }}+k_{\text {det }}(F)} \quad \text { (Equation 2) }
$$

where $k_{\text {attach }}$ is the force-independent attachment rate, described by:

$$
k_{\text {attach }}=\left(\frac{1}{k_{c a t}}-\frac{1}{k_{0}}\right)^{-1} \quad \text { (Equation 3) }
$$

The resulting force-dependent duty ratio is plotted in Fig. 4A. Here, we plot only resistive forces since the ensemble behavior of heart muscle is to move against a load (blood pressure) during contraction. Duty ratio is increased when myosin spends more of its catalytic cycle in the forceproducing state, bound to actin. Thus, mutant motors with faster detachment rates have lower duty ratios. In this case, the effect of reduced $\delta$ in pliant mutations had the strongest effect on duty ratio: even for $\mathrm{S} 782 \mathrm{~N}$, which only affected force sensitivity, duty ratio was lowered at resistive forces. For D778V, this effect was compounded by the marked increase in $k_{0}$, which was not offset by the relatively smaller increase in $k_{\text {cat }}$. For $L 781 \mathrm{P}$, the effect of reduced $\delta$ dominated the decreased $k_{0}$, except at high resistive force.

Using duty ratio, we can also calculate the average force that an individual myosin would exert in an ensemble system, which is again dependent on the load force ${ }^{15}$ :

$$
F_{a v}(F)=F r(F) \quad \text { (Equation 4) }
$$

Results from this calculation are plotted in Fig. 4B. The overall trends for average force resemble that of duty ratio because average force is just load force (the $\mathrm{x}$-axis) multiplied by duty ratio.

Finally, power output is a function of force $\times$ velocity. Velocity itself a function of detachment rate $k_{\text {det }}$ and step size $d$ :

$$
\operatorname{vel}(F)=k_{\text {det }}(F) \times d \quad(\text { Equation } 5)
$$


such that power output is calculated by:

$$
P_{a v}(F)=\operatorname{vel}(F) \times F_{a v}(F)
$$

(Equation 6)

Results from this calculation are plotted in Fig. 4C. In this case, the increased velocity of D778V myosin dominates over the reduced average force, resulting in a large increase in power output. For L781P, the reduction in both step size and detachment rate results in a decrease in power output. S782N, by contrast, is relatively similar to WT in its power output.
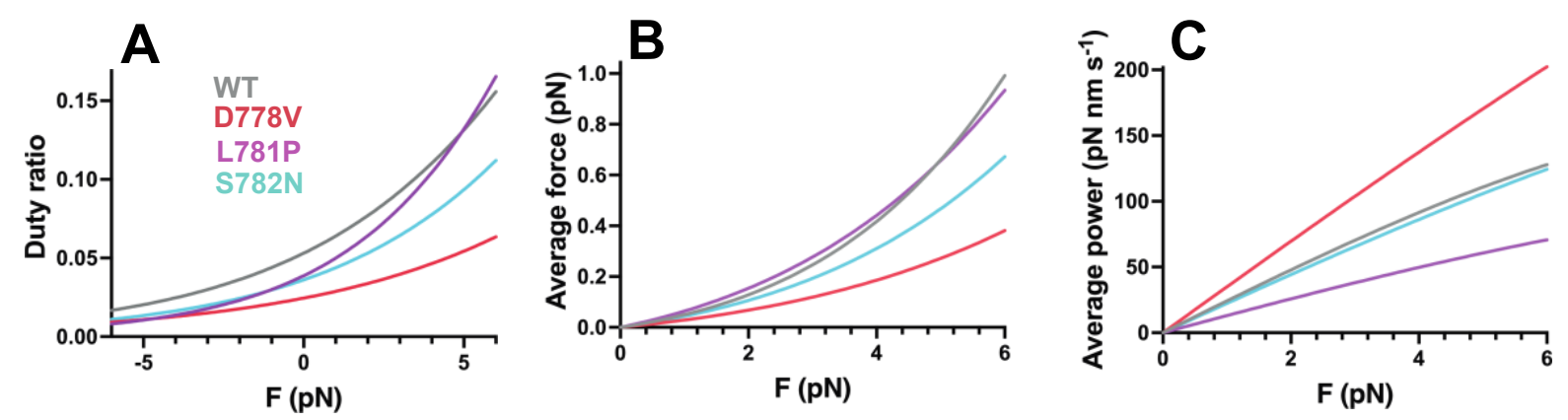

Figure 4: Effects of pliant region mutations on ensemble duty ratio, average force, and average power. Calculation of (A) duty ratio, $(B)$ average force, and (C) average power output at resistive forces based on measured detachment rate $k_{0}$, actin-activated ATPase rate $k_{\text {cat, }}$, force-dependent detachment rate $k_{\operatorname{det}}(F)$, and step size (see equations 2-6). WT curves are shown in gray, D778V curves are shown in red, L781P curves are shown in purple, and S782N curves are shown in cyan. In B, a representative dotted line is shown to demonstrate force if duty ratio was 1 .

\section{Lever arm mutants result in increased actin-activated ATPase when the $\mathbf{S} 2$ tail is present}

To assess whether lever arm mutations impact myosin S2 tail-based autoinhibition, we introduced the mutations into a 25-hep myosin construct that is identical to 2-hep myosin, except that it contains the first 25 heptads of the S2 tail, rather than only the first 2 heptads. Thus, the myosin tail is long enough in the 25-hep construct for the myosin heads to fold back onto the tail. We have previously shown that this 25 -hep myosin has a reduced $k_{\text {cat }}$ as compared to the 2-hep myosin, such that a significant proportion of the 25-hep myosin is autoinhibited by the presence of the $S 2$ tail $^{10,34}$. This autoinhibition could arise from the IHM structure, where some of the myosin 
heads are sterically unable to bind actin. Mutations that disrupt autoinhibition show smaller differences between the 2-hep and 25-hep $k_{\text {cat }} s^{11-13}$.

Here, we found that all five of the lever arm mutations showed smaller differences between 2-hep and 25-hep as compared to WT (Fig. 5, see full values in table S1). A797T had the most dramatic result, where the 25 -hep myosin showed an apparent increase in $k_{\text {cat }}$ as compared to 2 -hep. The other four mutations resulted in smaller differences between 2- and 25-hep myosin, with reductions comparable to some previous mutations we have measured (D382 $Y^{11}$ and $R 403 Q$ and $\left.\mathrm{R} 663 \mathrm{H}^{12}\right)$.
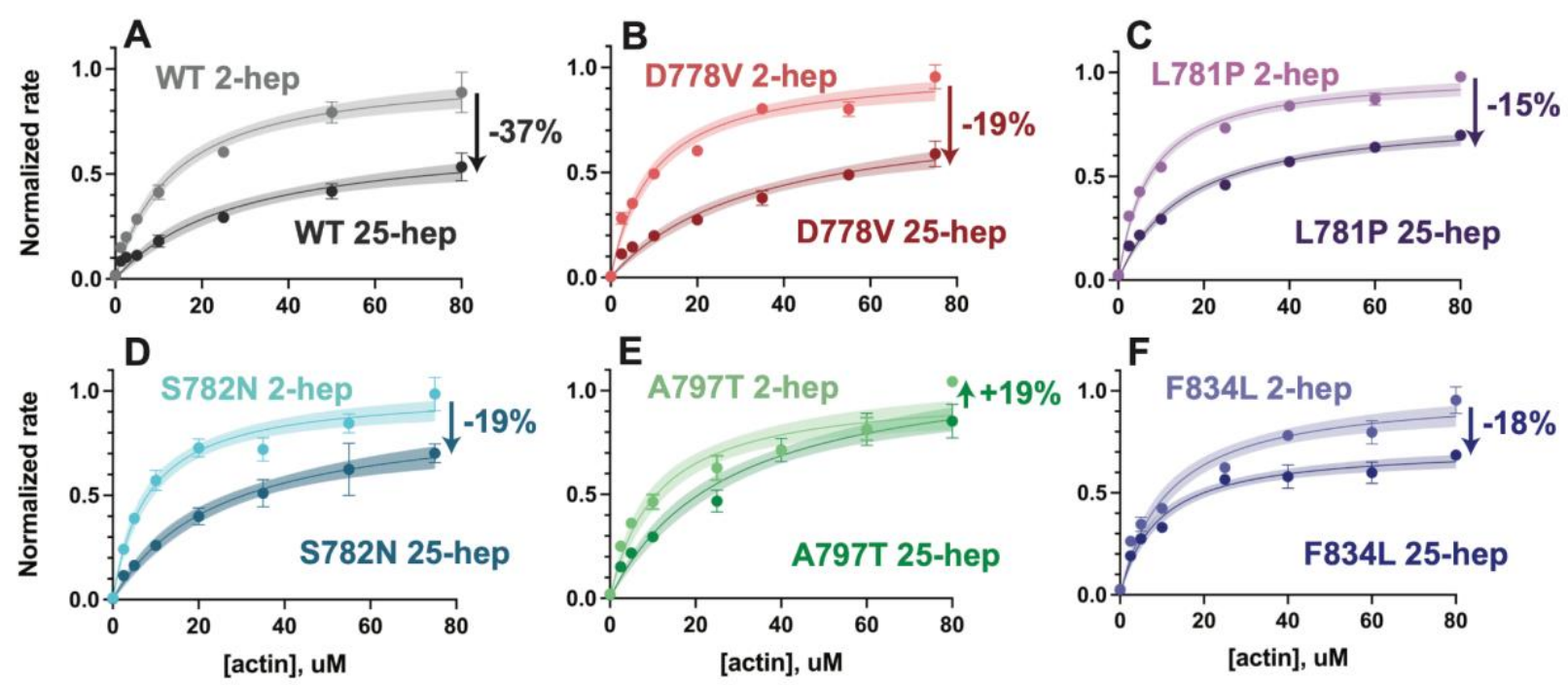

Figure 5: Actin-activated ATPase rates of 2-hep vs. 25-hep $\beta$-cardiac myosin constructs. A-F) Representative actin-activated ATPase curves for each mutant, normalized to the mutant 2-hep control $k_{\text {cat. }}$ A) is reproduced from $\left[{ }^{13}\right]$. Each data point represents the average of three technical replicates of one biological replicate (one of two, see table S1), and error bars represent standard deviations. Where error bars are not shown, error is smaller than the size of the data point. Curves are fitted to Michaelis-Menten kinetics, and shaded areas represent the $95 \%$ confidence intervals of the fits. Arrows with percentages on each graph show the percent change from the 2-hep to the matched 25-hep (average of both biological replicates, not just the representative curve shown). See table $\mathrm{S} 1$ for full results.

\section{Mutations in the light chain binding domain reduce SRX, while pliant region mutations do not reduce SRX}

To determine the effect of lever arm mutations on the super relaxed state, we next measured the single-turnover ATPase activity of the myosins in the absence of actin. In this experiment, myosin 
is loaded with a roughly equimolar amount of fluorescent mant-ATP, then chased with excess unlabeled "dark" ATP $24,31,33$. The fluorescence of the sample decays, and the decay rate can only be adequately fit with a double-exponential function (it does not fit a single exponential), suggesting the presence of two distinct structural states. We have previously correlated the slower SRX rate to a folded back state, whereas the faster DRX rate appears to be correlated with a structure where the heads are not bound to the $\mathrm{S} 2$ tail $^{24,33}$.

Here, we performed the single-turnover ATPase experiment with mutant and WT 25-hep myosins. Surprisingly, the pliant region mutations showed no significant reduction in the SRX state (Fig. $6 \mathrm{~A}-\mathrm{D}, \mathrm{G} ; \mathrm{WT}=57 \pm 10 \%($ mean $\pm \mathrm{SD})$; D778V = $63 \pm 10 \%, \mathrm{p}=0.20 ; \mathrm{L} 781 \mathrm{P}=61 \pm 11 \%, \mathrm{p}=$ 0.42 ; and $S 782 \mathrm{~N}=68 \pm 5 \%, p=0.018)$. In fact, $S 782 \mathrm{~N}$ showed a statistically significant, though functionally small, increase in the SRX phase. This unanticipated result is in opposition to the actin-activated ATPase result comparing the 2- vs. 25-hep constructs, which suggested that the autoinhibition was disrupted. In contrast, the light chain-binding region mutations did show a statistically significant reduction in SRX compared to WT (Fig. 6E-F, G; A797T = $26 \pm 6 \%$, a 31\% decrease, $p<0.0001$; and F834L $=16 \pm 4 \%$, a 41\% decrease, $p<0.0001$ ), in keeping with their increases in 25-hep actin-activated ATPase rates. None of the mutations affected the rates of either the fast or slow phases (table S2).
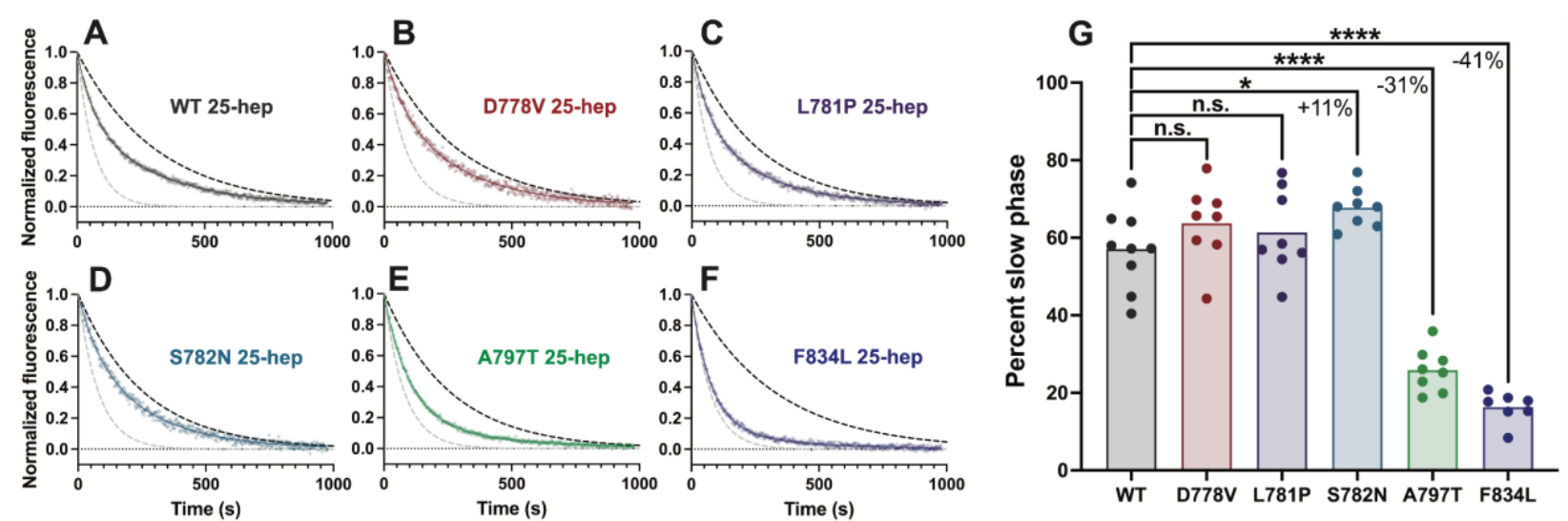

Figure 6: Single ATP turnover kinetics for 25-hep myosin lever arm mutants compared to WT. A-F) Representative single mant-ATP turnover curves for WT 25-hep and each mutant. Each curve is fitted to a bi-exponential decay and normalized to the fitted $Y_{0}=1.0$ and plateau value $=0.0$. The dotted black line represents a simulated single-exponential decay with the slow rate of the plotted data, and the dotted gray line represents a simulated single-exponential decay with the fast rate of the plotted data. G) Percent slow phase of multiple replicates of each mutant. Pairwise comparisons show that only A797T and F834L 
significantly reduce the SRX state, resulting in a $31 \pm 4 \%$ and $41 \pm 4 \%$ (mean $\pm \mathrm{SEM}$ ) reduction in slow phase, respectively. S782N resulted in an $11 \pm 4 \%$ (mean \pm SEM) increase in SRX. * indicates $p \leq 0.05$, ${ }^{* * * *}$ indicates $p \leq 0.0001$

As a control, we assayed the single-turnover ATPase rates of the 2-hep constructs, and we did not see any statistically significant changes in the percent SRX turnover for any of the mutations as compared to WT 2-hep (Fig. S2; WT = $21 \pm 5 \%$; D778V = $16 \pm 9 \%, p=0.19$; L781P = $23 \pm$ $2 \%, p=0.23 ; S 782 N=21 \pm 4 \% ; p=0.99 ; A 797 T=18 \pm 6 \%, p=0.08 ; F 834 L=26 \pm 5 \%, p=0.12$ (mean \pm SD)). This suggests that the increased SRX turnover for both WT and the pliant mutations is S2 tail-dependent, and not based on any changes specific to the motor domain. In contrast, the A797T and F834L 2-heps had a similar fraction of SRX turnover as compared to the 25-hep constructs, suggesting that these mutations virtually extinguish the S2 tail-dependent autoinhibition measured by the single-turnover assay.

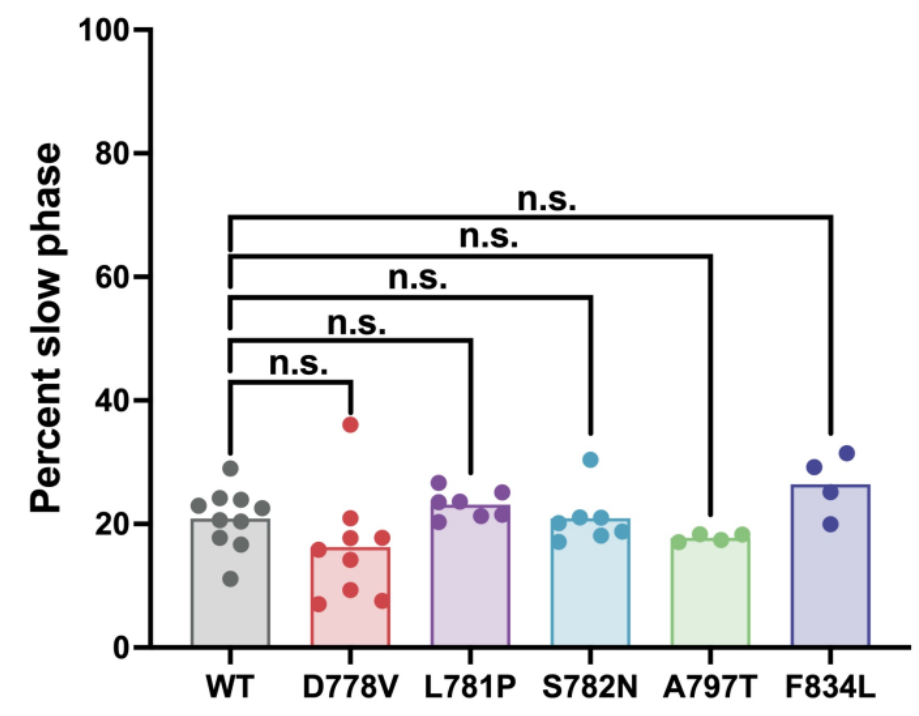

Figure S2: Single ATP turnover kinetics for WT vs. mutant 2-hep myosins. Percent slow phase for 2hep myosins. For all mutations, the slow phase is similarly low as compared to the WT control. 


\begin{tabular}{|c|c|c|c|c|c|c|c|c|}
\hline Protein & $N$ & $\begin{array}{c}\text { Average } \\
\text { percent fast } \\
\text { phase }\end{array}$ & $\begin{array}{c}\text { Average } \\
\text { percent } \\
\text { slow phase }\end{array}$ & $\begin{array}{c}\text { Percent fast } \\
\text { and slow } \\
\text { phase SD }\end{array}$ & $\begin{array}{c}\text { Average } \\
\text { fast rate, } \mathrm{s}^{-1}\end{array}$ & $\begin{array}{c}\text { Fast rate } \\
\text { SD, } \mathbf{s}^{-1}\end{array}$ & $\begin{array}{c}\text { Average } \\
\text { slow rate, } \\
\mathbf{s}^{-1}\end{array}$ & $\begin{array}{c}\text { Slow rate } \\
\text { SD, } \mathbf{s}^{-1}\end{array}$ \\
\hline WT 25-hep & 9 & $42.9 \%$ & $57.1 \%$ & $10.3 \%$ & 0.0185 & 0.0121 & 0.00316 & 0.00055 \\
\hline D778V 25-hep & 8 & $36.3 \%$ & $63.7 \%$ & $10.0 \%$ & 0.0220 & 0.0217 & 0.00342 & 0.00040 \\
\hline L781P 25-hep & 8 & $38.6 \%$ & $61.4 \%$ & $11.0 \%$ & 0.0154 & 0.0057 & 0.00360 & 0.00027 \\
\hline S782N 25-hep & 8 & $32.2 \%$ * & $67.8 \%{ }^{*}$ & $5.2 \%$ & 0.0134 & 0.0042 & 0.00328 & 0.00073 \\
\hline A797T 25-hep & 8 & $74.1 \% \%^{* * * *}$ & $25.9 \%$ **** & $5.6 \%$ & 0.0113 & 0.0010 & 0.00254 & 0.00074 \\
\hline F834L 25-hep & 7 & $83.7 \%$ **** & $16.3 \%$ **** & $4.0 \%$ & 0.0135 & 0.0006 & 0.00270 & 0.00086 \\
\hline WT 2-hep & 10 & $79.1 \%$ & $20.9 \%$ & $4.9 \%$ & 0.0132 & 0.0018 & 0.00196 & 0.00095 \\
\hline D778V 2-hep & 9 & $86.2 \%$ & $16.3 \%$ & $8.9 \%$ & 0.0135 & 0.0016 & 0.00303 & 0.00155 \\
\hline L781P 2-hep & 7 & $76.9 \%$ & $23.1 \%$ & $2.3 \%$ & 0.0135 & 0.0030 & 0.00167 & 0.00105 \\
\hline S782N 2-hep & 7 & $79.1 \%$ & $20.9 \%$ & $4.4 \%$ & 0.0150 & 0.0020 & 0.00294 & 0.00108 \\
\hline A797T 2-hep & 4 & $82.2 \%$ & $17.8 \%$ & $0.6 \%$ & 0.0134 & 0.0007 & 0.00184 & 0.00046 \\
\hline F834L 2-hep & 4 & $73.6 \%$ & $26.4 \%$ & $5.0 \%$ & 0.0153 & 0.0016 & 0.00253 & 0.00148 \\
\hline
\end{tabular}

Table S2: Full single ATP turnover results. Single ATP turnover experiments were performed for both 2and 25-hep myosins. Results show $N$, the number of independent experiments for each protein, average percent fast and slow phase $\pm S D$, and average fast and slow rates \pm SD. Stars denote statistically significant changes as compared to the WT control of the same protein construct, where ${ }^{*}$ indicates $p \leq$ 0.05 and $^{* * * *}$ indicates $p \leq 0.0001$. 


\section{DISCUSSION}

The myosin lever arm performs one of the most important functions of the myosin chemomechanical cycle: it amplifies the transduction of the chemical energy of ATP hydrolysis into physical motion, allowing for myosin's motor function. This crucial role explains why the lever arm is both highly conserved and a hotspot for HCM-causing mutations. Here, we have characterized five such mutations spanning the pliant region and the light chain-binding regions, giving insights into the role of the lever arm in generating force and power output, as well as its role in myosin S2 tail-based autoinhibition. The impacts of the mutations on myosin function segregate them naturally into two categories: the light chain-binding region mutations, A797T and F834L, behave very differently from the pliant region mutations D778V, L781P, and S782N.

The functional changes in myosin's motor activity caused by the pliant region mutations were dramatic and ran the full spectrum of potential outcomes: D778V increased actin-activated ATPase of the 2-hep construct along with in vitro motility velocity and $k_{0}$, L781P decreased motility velocity, $k_{0}$, and step size, and S782N did not result in changes in any parameter except the force sensitivity of the detachment rate $\delta$. That these mutations can have such varied and substantial effects on myosin activity speaks to the importance of the pliant region in coupling the activity of the lever arm to the rotation of the converter domain.

One feature that was shared amongst the pliant region mutations was a reduction in the force sensitivity $\delta$. Reduced force sensitivity suggests a mechanism where mutations partially uncouple the force perceived at the ATP binding site from the force applied at the anchor point at the Cterminus of the lever arm. We previously observed a decrease in $\delta$ for the P710R mutation, which is located in between the $\mathrm{SH}$ helices and the converter domain approximately $20 \AA$ from the pliant region ${ }^{13}$. Pliant region mutations may likewise reduce rigidity, thus uncoupling the force transduction and reducing $\delta$. The term "pliant region" itself comes from the varied positions of this region observed in different crystal structures ${ }^{37,53}$, suggesting that its compliance or ability to assume multiple conformations is an important feature of its function; thus, any alteration to the rigidity of this region would likely have strong impacts on myosin function in sarcomeres. It is possible that reductions in $\delta$ as a result of pliant region mutations could impact contraction and/or relaxation kinetics in patients with these specific mutations. 
Additional, divergent changes resulting from the pliant region mutations may be a function of the identities of the mutations themselves. For example, the L781P mutation introduces a helixbreaking proline into a continuous $\alpha$-helix. If the lever arm alpha helix is destabilized, this could explain the observed decrease in step size for the L781P mutation: a more bent lever arm would generally produce a lower step size. Additionally, the D778V mutation replaces a highly polar amino acid with a more hydrophobic one. This mutation dramatically increases detachment rate, suggesting that amino acids in the pliant region, which are very distant from the actin-binding domain, can play a role in the highly allosteric process of actin detachment. Conversely, the S782N mutation has comparatively few impacts on the kinetics of the myosin motor, suggesting that some mutations in the pliant region may be well tolerated in the context of motor domain function.

The sum total of the effects of pliant region mutations on motor activity (outside of potential impacts on autoinhibition) is reflected in the changes in calculated average power output (Fig. $4 \mathrm{C})$, which takes into account actin-activated ATPase rate $k_{\text {cat }}$, force-dependent detachment rate $k_{\operatorname{det}}(F)$, force-independent detachment rate $k_{0}$, and step size $d$. For the pliant region mutations, D778V appears to be hypercontractile, L781P appears to be hypocontractile, and S782N is very similar to WT (although very slightly hypocontractile). A prominent model in the field suggests that HCM mutations increase myosin activity in ensemble, resulting in hypercontractility at the cellular level ${ }^{14}$. The magnitude of the increases in D778V's actin-activated ATPase activity and motility velocity were comparable to the early-onset mutations $\mathrm{D} 239 \mathrm{~N}$ and $\mathrm{H} 251 \mathrm{~N}^{8}$ and not far from the changes observed for 1457T, which had the largest changes we've observed to date ${ }^{11}$. Thus, it is possible that these increases alone could lead to hypercontractility in a cellular context. However, the same cannot be said of L781P and S782N, where a different rationale would have to apply, given that these mutations resulted in either decreased or no change in contractility, respectively. Thus, we additionally considered the possibility that these mutations might impact myosin S2 tailbased autoinhibition.

The pliant region mutations showed a unique phenotype where they had a smaller reduction between the actin-activated ATPase rate of the 2-hep vs. the 25-hep constructs as compared to WT, suggesting reduced autoinhibition, but did not show any statistically significant changes in the SRX/DRX ratio in the single turnover ATPase assay. We have used these two assays to study a number of mutations to date ${ }^{11-13}$, and all previously studied mutations had shown concordant behavior in these two assays. One way to interpret this data is that the actin-activated ATPase of 
25-hep suggests that autoinhibition is disrupted by the mutations, but only in the presence of actin, such that the single turnover ATPase is unchanged. Previous data supports a model where the presence of actin can influence and disrupt myosin autoinhibition ${ }^{33}$, so it stands to reason that mutations could specifically affect this activity. This model would suggest that in the in vivo context, myosin activity would be increased specifically during contraction when actin is available for myosin binding, thus increasing force production, but during relaxation when actin-binding is blocked by tropomyosin, there would be the same proportion of SRX compared to WT. It is clear from structures of smooth muscle myosin in the folded state that the conformation of the pliant region is different in the two heads ${ }^{42,43}$ : the blocked head pliant region takes on a bent conformation, while in the free head pliant region is much straighter. Thus, logically, mutations could destabilize the IHM by changing the available positions of the pliant region, leading to the reduced autoinhibition we observed and thus hypercontractility. Alternatively, it is possible that this study of the pliant region mutations may be limited by isolating the myosin outside of the context of sarcomeres, where structural constraints or additional proteins (such as myosin binding protein-C) could impact myosin activity and/or the formation of the autoinhibited state. Regardless, future experiments introducing pliant region mutations into a cardiomyocyte model would be useful for helping to test whether these mutations indeed result in net hypercontractility at the cellular level, and if so, how that would arise and lead to cellular hypertrophy.

In contrast to the pliant region mutations, the light chain-binding region mutations both had very little or no impact on myosin's in vitro motility velocity or actin-activated ATPase activity of the 2hep construct. However, both mutations had clear impacts on myosin's S2 tail-based autoinhibition, reflected by both an increase in the $S 2$ tail-containing 25-hep actin-activated ATPase rate and a decrease in the proportion of SRX. Thus, it is likely that the light chain-binding regions of the lever arm play an important role in myosin $\mathrm{S} 2$ tail-based autoinhibition. Most myosin mutations that have been investigated in the context of myosin autoinhibition have been in regions thought to be directly involved in forming structurally necessary contacts for the IHM state, including mutations in both the myosin "mesa" region and the S2 tail ${ }^{10,11}$, which likely interact with each other in the IHM, as well as mutations at the putative head-head interface ${ }^{12}$. In contrast, A797T and F834L do not appear to be involved in such contacts in modeled structures of $\beta$ cardiac myosin in the IHM state ${ }^{10,22,23}$. Thus, if the defects in autoinhibition that we observed for A797T and F834L indeed indicate reductions in the IHM structural state, these mutations are likely to affect the IHM structure by an allosteric mechanism. A simple model could be that the A797T and F834L mutations affect the position or rotation of the light chains about the lever arm. While 
our data shows that the light chains were still able to load onto the lever arm at the proper ratios, it is possible that specific light chain positioning is required to access the folded state. Indeed, in structures of smooth muscle myosin in the folded state ${ }^{42}$, it was shown that the RLC in particular took a position such that its phosphorylation sites were very close together in the IHM state, which the authors hypothesized created energetically unfavorable charge-charge interactions when the sites were phosphorylated (phosphorylation has been shown to move heads out of the autoinhibited state). Thus, positioning of the light chains appears to be important for forming the folded state.

Alternatively, it is possible that the light chain-binding region mutations do not affect the positioning of light chains; instead, they could affect the structure of the lever arm itself in ways that prevent autoinhibition. It is apparent from the modeled structures of $\beta$-cardiac myosin in the IHM state that the lever arm may form an unusual conformation to allow the heads to fold back. In all three structures, the myosin heads and S2 tails overlay remarkably well; however, the lever arms vary dramatically ${ }^{10,22,23}$ (Fig. S3A-C). Notably, in all three modeled structures, the lever arm's alpha helix is broken; this differs from known structures of the folded state in other myosins, where the alpha helix is largely unbroken ${ }^{42,43}$. Thus, it is possible that a specific or unusual conformation of the lever arm might be required to allow the IHM structure, and mutations might preclude specifically those conformations. For example, in molluscan myosin, where the folded back state has been extensively studied, the lever arm plays a key role in formation of the folded back state, and a few regions in particular have been identified as important. The first is the slightly bent region of the lever arm directly in between the two light chains; in molluscan myosin, this is where $\mathrm{Ca}^{2+}$ directly binds to myosin and activates it from the folded back autoinhibited state ${ }^{38}$. Coincidentally, the A797T mutation is very near to that slight bend (Fig. S3D). Another region that has been identified as important in molluscan myosin is the "hook" or "ankle" joint near the Cterminus of the lever $\operatorname{arm}^{40,41}$. This feature is common across the myosin II class and can bend to very different angles, which is thought to help allow for the folded back conformation. The F834L mutation is very close to that ankle joint, and thus could affect the conformation about that joint (Fig. S3E). In any case, a high-resolution structure of human $\beta$-cardiac myosin in the IHM state will be useful to further understand the structural requirements of the lever arm for autoinhibition. 


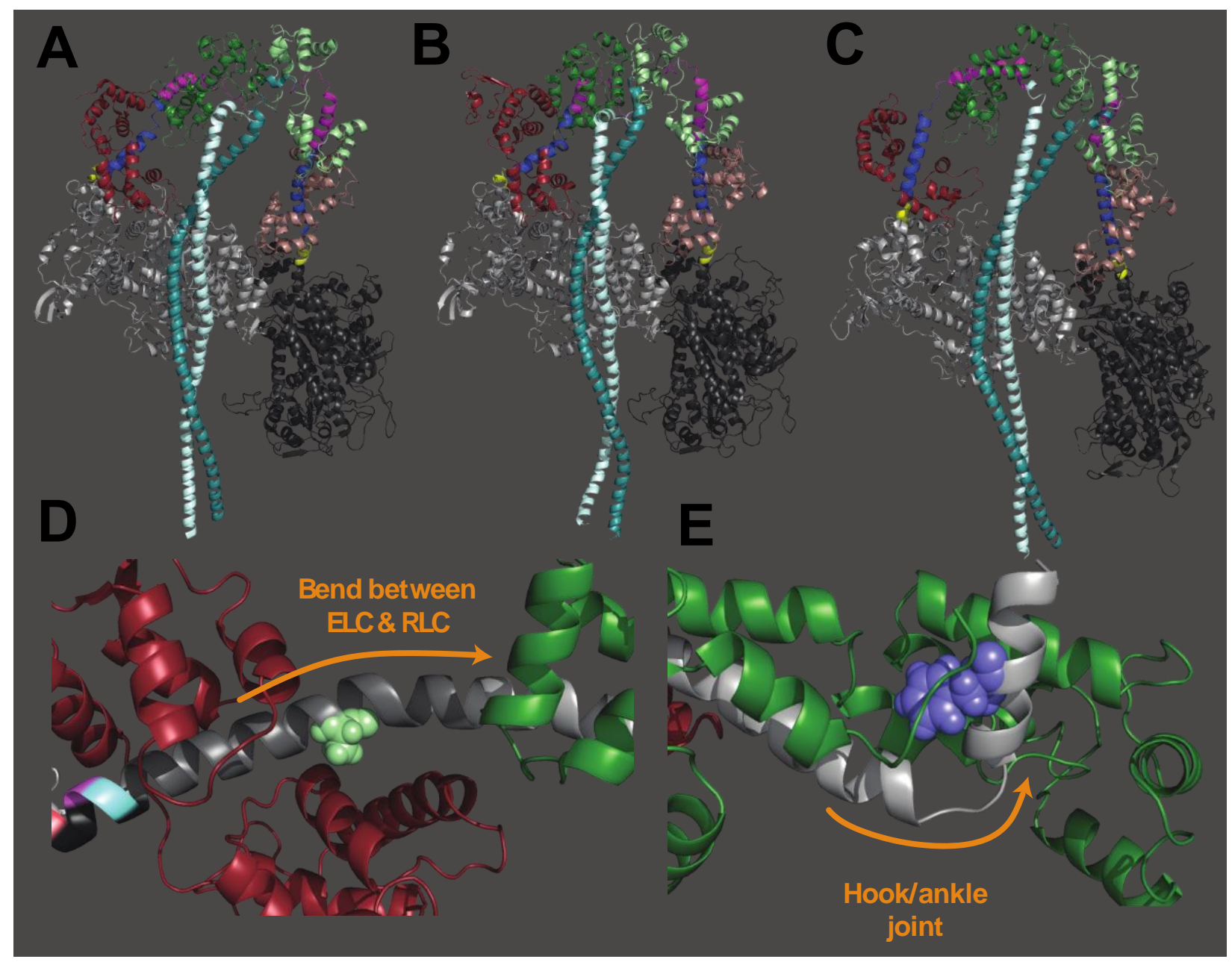

Figure S3: Contributions of lever arm position to the folded state structure. A-C) Homology modeled structures of human $\beta$-cardiac myosin in the IHM structural state from A) [22], B) [10], and C) [23]. In each structure, the pliant region is colored yellow, the ELC binding region is colored blue, and the RLC binding region is colored pink. The myosin heads are in gray, ELCs are in dark red and brown, RLCs are in green, and S2 tail regions are in cyan. D) A797 (light green) is located in the region where the lever arm bends between the ELC and RLC. This region has been previously implicated in formation of the folded state. E) F834 (blue) is located very near to the hook or ankle joint at the end of the lever arm. This joint has been previously shown to be important in autoinhibition. D) and E) are from a homology modeled structure of human $\beta$-cardiac myosin in the pre-stroke state ${ }^{48}$ (see Fig. 1A).

In summary, this study allowed for a detailed analysis of lever arm function, specifically focusing on how HCM-causing mutations in both the pliant and light chain-binding regions of the lever arm alter myosin activity. While the light chain-binding region mutants clearly showed a phenotype of reducing myosin autoinhibition, potentially by disrupting the folded back IHM conformation, the 
pliant region mutants had a more complicated phenotype that begs further investigation. With a high density of mutations across a very small region of myosin, the pliant region seems to play an intriguing role in both transducing force from the motor domain to the lever arm and potentially forming the IHM structure. Future research into the lever arm region, particularly using in vivo models, could further clarify the role of the lever arm in both force transduction and formation of the folded state. 


\section{MATERIALS AND METHODS}

\section{Protein expression and purification}

Recombinant human $\beta$-cardiac myosin constructs described within, including short subfragment S1 (sS1), 2-hep (short-tailed), and 25-hep (long-tailed), were purified as described previously ${ }^{10,54}$ with some minor modifications. Heavy chain myosin (MYH7) was co-expressed with human essential light chain (ELC) (MYL3) containing an N-terminal FLAG tag followed by a TEV protease site in differentiated mouse myoblast (C2C12) cells using adenovirus vectors from the AdEasy Vector System (Qbiogene Inc., Carlsbad, California, USA). The sS1 construct used in this study contained a C-terminal eGFP tag, while the 2-hep and 25-hep constructs contained both eGFP and PDZ C-peptide on their $\mathrm{C}$ termini, respectively. C2C12 cells were infected with adenovirus constructs 48 hours after differentiation and harvested 4 days after infection in a lysis buffer containing $50 \mathrm{mM} \mathrm{NaCl}, 20 \mathrm{mM} \mathrm{MgCl}$, $20 \mathrm{mM}$ imidazole $\mathrm{pH}$ 7.5, 1 mM EDTA, 1 mM EGTA, 1 mM DTT, 3 mM ATP, 1 mM PMSF, 5\% sucrose, and Roche protease inhibitors. Cells were then immediately flash frozen in liquid nitrogen. Note that this lysis buffer composition differs from our previously published methods-salt concentration and sucrose are lowered and $\mathrm{MgCl}_{2}$ concentration is raised to encourage the native mouse myosin to form filaments, reducing contamination. Pellets were stored up to 6 months at $-80^{\circ} \mathrm{C}$, then thawed at room temperature for purification.

Cells were lysed with 50 strokes of a dounce homogenizer and clarified by spinning at 30,000 RPM in a Ti-60 fixed angle ultracentrifuge rotor for 30 minutes. Supernatant was bound to antiFLAG resin for $1-2$ hours at $4^{\circ} \mathrm{C}$. The resin was then washed with a wash buffer containing 150 $\mathrm{mM} \mathrm{NaCl}, 5 \mathrm{mM} \mathrm{MgCl}$, $20 \mathrm{mM}$ imidazole $\mathrm{pH}$ 7.5, 1 mM EDTA, 1 mM EGTA, 1 mM DTT, 3 mM ATP, $1 \mathrm{mM}$ PMSF, 10\% sucrose, and Roche protease inhibitors. For both the 2- and 25-hep constructs, native mouse RLC was depleted with a buffer containing $20 \mathrm{mM}$ Tris pH 7.5, $200 \mathrm{mM}$

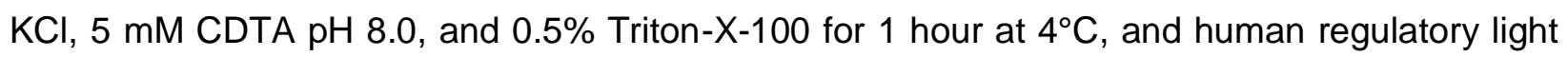
chain (RLC) (purified from E. coli as previously described ${ }^{10}$ ) was added to the resin with wash buffer and incubated for $>2.5$ hours at $4^{\circ} \mathrm{C}$. The resin was then incubated overnight with TEV protease at $4^{\circ} \mathrm{C}$ to cleave the ELC-myosin complex off of the resin. The next day, the supernatant was further purified using a HiTrap Q HP column on an FPLC with a gradient of 0-600 mM NaCl over 20 column volumes in a buffer containing $10 \mathrm{mM}$ imidazole $\mathrm{pH} 7.5,4 \mathrm{mM} \mathrm{MgCl}_{2}, 10 \%$ sucrose, $1 \mathrm{mM}$ DTT, and $2 \mathrm{mM}$ ATP. Pure fractions (determined by Coomassie staining on 10\% SDS PAGE) were collected and concentrated to 5-50 uM using Amicon Ultra 0.5 mL centrifugal 
filters with a 50 or $100 \mathrm{kDa}$ cutoff, which aids in removing any unbound ELC or RLC. The myosin was then used directly for ATPase assays, buffer exchanged for single turnover assays (described below), or flash frozen in liquid nitrogen for in vitro motility or optical trapping experiments.

\section{Deadheading}

For further analysis using in vitro motility or optical trapping, the myosin was subjected to a "deadheading" procedure to remove any myosin that bound irreversibly to actin. After thawing on ice, myosin was first incubated with a $>10 \mathrm{X}$ excess of F-actin for 5 minutes on ice. 2 mM ATP was then added to the mixture, and it was further incubated for 3 minutes on ice. The $F$-actin was then pelleted by ultracentrifugation at 95K RPM in a TLA-100 rotor, and the supernatant containing active myosin was collected and used. This procedure was applied to all 2-hep myosin samples used for in vitro motility, while we found empirically that it was only necessary to deadhead the D778V sS1 myosin for optical trapping (the WT and other mutants were not deadheaded). This is because in a single molecule experiment, a "dead" myosin head does not generate useful data; instead, it binds to the actin irreversibly and destroys the actin dumbbell. This rarely occurred for the WT, L781P, and S782N myosins, but was more frequent for D778V. Thus, we deadheaded only D778V to reduce dumbbell loss.

\section{In vitro motility}

Motility measurements of 2-hep WT and mutant myosins were collected as described previously ${ }^{49}$. Multi-channel flow chambers were constructed on microscopy slides using doublesided tape and coverslips coated in $0.1 \%$ nitrocellulose $/ 0.1 \%$ collodion in amyl acetate. SNAPPDZ (purified from E. coli as described previously ${ }^{49}$ ) was first flowed into each channel at a concentration of $3 \mathrm{uM}$ in assay buffer (AB: $25 \mathrm{mM}$ imidazole $\mathrm{pH}$ 7.5, $25 \mathrm{mM} \mathrm{KCl}, 4 \mathrm{mM} \mathrm{MgCl}, 1$ $\mathrm{mM}$ EGTA, and $10 \mathrm{mM}$ DTT) and incubated for 2 minutes at room temperature. Then, each channel was blocked with assay buffer plus $1 \mathrm{mg} / \mathrm{mL}$ BSA (ABBSA) twice for 2 minutes each. Myosin, diluted to 50-100 nM in ABBSA, was then flowed into each channel and incubated for 2 minutes. Each channel was then washed with ABBSA, then loaded with final GO buffer containing 2 mM ATP, 2.5 nM TMR-phalloidin-labeled actin, and an oxygen-scavenging system $(0.4 \%$ glucose, $0.11 \mathrm{mg} / \mathrm{mL}$ glucose oxidase, and $0.018 \mathrm{mg} / \mathrm{mL}$ catalase) in ABBSA.

The slide was then imaged on a Nikon Ti-E inverted microscope with a 100x TIRF objective at a rate of $2 \mathrm{~Hz}$ with an exposure of $300 \mathrm{~ms}$ for 30 seconds on an Andor iXon+EMCCD camera. Each 
channel was imaged in three separate frames, and that data was combined for analysis. Movies were analyzed using FAST (Fast Automated Spud Trekker) ${ }^{49}$ for filament tracking and velocity measurement using the following parameters: window size $n=5$, path length $p=10$, percent tolerance $p t=20$, and minimum velocity for stuck classification $\min v=80 \mathrm{~nm} / \mathrm{s}$. Filtered mean velocity was used as unloaded velocity. Temperature varied from $21-23^{\circ} \mathrm{C}$ during imaging; these temperature variations result in some variation in velocity between slides, but every mutant channel was imaged on the same slide at the same temperature as a WT control. Each mutant was then normalized to the WT control from the same slide, which negates the effect of slight variations in temperature.

\section{Optical trapping}

To obtain load dependent detachment rates of single myosin-actin interaction events, harmonic force spectroscopic (HFS) measurements were performed in a dual-beam optical trap. The details of the instrumental setup and the method are similar to what has been previously described ${ }^{15,50,51}$. The sample chambers for this experiment are made by sticking a No. 1.5 coverslip onto a $1 \mathrm{~mm}$ thick microscope slide with the use of double-sided tape (Fig. S4A, bottom). These coverslips are spin-coated with silica beads (diameter 1.6 micrometer, Bangs Laboratories, Fishers, IN, USA) and then with an amyl acetate solution containing $0.1 \%$ nitrocellulose and $0.1 \%$ collodion. After forming the chamber, the surface of the coverslip and the platform beads are functionalized by flowing anti-GFP antibody (product \#ab1218, Abcam, Cambridge, United Kingdom) solution in AB buffer (25 mM imidazole at pH 7.5, $25 \mathrm{mM} \mathrm{KCl,} 4 \mathrm{mM} \mathrm{MgCl}, 1 \mathrm{mM}$ EGTA, and $10 \mathrm{mM}$ DTT) into the chamber for 2-3 minutes. The concentration of the anti-GFP antibody solution is kept low (1 $\mathrm{nM})$ to ensure only a stochastic presence of the molecules on the surface. BSA $(1 \mathrm{mg} / \mathrm{mL})$ solution in $A B$ buffer ( $A B B S A$ ) is then flown into the chamber for 2-3 minutes to passivate/block the remaining uncovered glass surfaces. A $50 \mathrm{nM}$ solution of GFP functionalized (C-terminal) short construct of either WT or mutants of human $\beta$-cardiac myosin (sS1-eGFP) in ABBSA buffer is then flown into the chamber for 2-3 minutes. These sS1-eGFP molecules are thus translationally immobilized on the surface by binding to the anti-GFP antibody (Fig. S4A, top). The unattached free myosin molecules are then washed away with ABBSA buffer. Finally, a solution containing filaments of $0.3 \mathrm{nM}$ TMR-phalloidin-labeled biotinylated actin (Cytoskeleton, Denver, CO, USA), $0.4 \%$ glucose, $0.11 \mathrm{mg} / \mathrm{mL}$ glucose oxidase, $0.018 \mathrm{mg} / \mathrm{mL}$ catalase, $2 \mathrm{mM} \mathrm{ATP}$, and 1 micrometer-diameter streptavidin-coated polystyrene beads (Bangs Laboratories) diluted ( 5000 times) in ABBSA is flown in, prior to the sealing of the chamber with vacuum grease. This entire sample chamber preparation is done at $23^{\circ} \mathrm{C}$. 

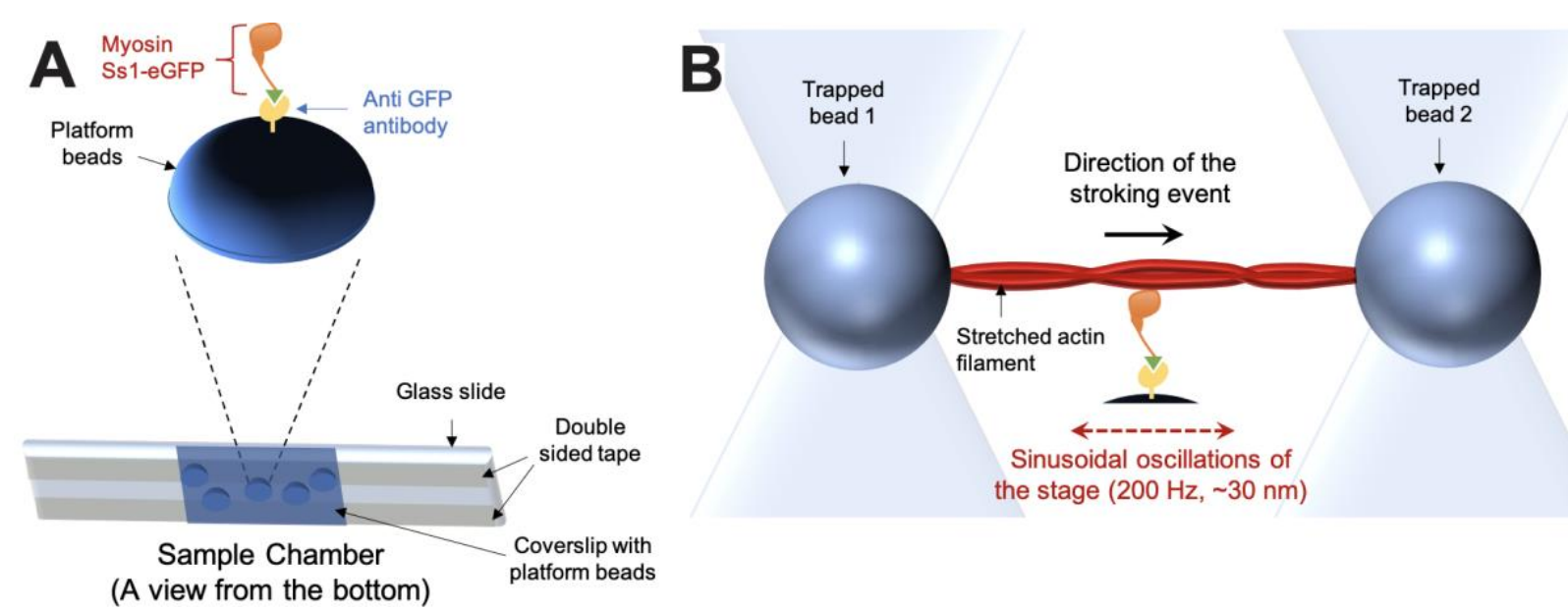

Figure S4: Technical details of the dual beam optical trap experiment. A) The sample chamber (bottom) is shown in an orientation suitable for an inverted microscope. On top, the typical arrangement of the protein complexes on top of a platform bead is depicted. B) A typical stroking event-as expected during actin-myosin interaction in a HFS setup-is depicted. In a standard HFS experiment, the stage oscillates, resulting in a variety of assistive or resistive external load forces applied to the stroking myosin.

The stiffnesses of the two optical traps were kept between 0.08-0.10 pN/nm for all experiments. Each trap was calibrated by trapping single polystyrene beads in them and then by using the power spectrum method, as described elsewhere ${ }^{15,50,51}$. Corrections were made to rectify the effect of the anti-aliasing filter in the system. The contributions from the surface effects were also corrected during calibration. At this point in the experiment-after calibration-each trap contained one streptavidin-coated polystyrene bead. Next, an actin filament was snared between the two trapped beads using a biotin-streptavidin linkage by moving the chamber (i.e. the microscope stage) with respect to the position of the optical traps in 3D. The filament is then stretched by pulling the two beads from their ends (by steering the optical traps away from each other) to form a "dumbbell" (Fig. S4B). At this point in the experiment, the actin filament is stretched and held in the solution. On the surface, the myosin molecules (in the presence of ATP) are ready to initiate stroking events upon actin interaction. The actin dumbbell is then lowered towards the platform beads on the surface (keeping the stage under $200 \mathrm{~Hz}$ oscillation) anticipating potential interactions with single myosin molecules (Fig. S4B). The stage oscillation imparts different amounts of assistive or resistive external forces during each stroking event based on the stochastic binding of myosin. In the time-trace data, the myosin-actin interactions 
and stroking events are identified by an expected change in the phase and the amplitude of the bead-oscillation in both the traps ${ }^{15}$. Upon detachment after the stroking events, the oscillation in the position of the two trapped beads returns to its initial value. Several stroking events for each myosin molecule are recorded. These stroking events are then identified and binned based on the extent of external force. Then, using maximum likelihood estimation, detachment rates for every force range are obtained from the durations of the events for each molecule ${ }^{15}$. The external load/force $(F)$ dependent change in the detachment rates $\left(k_{d}\right)$ is exponential in nature:

$$
k_{d}(F, \Delta F)=k_{0} I_{0}\left(\frac{\Delta F \delta}{k_{B} T}\right) e^{\frac{-F \delta}{k_{B} T}}
$$

Where $k_{B}$ is the Boltzmann constant, $T$ is temperature, $k_{0}$ is the detachment rate at zero external force, $I_{0}$ is a correction for the harmonic force with $\Delta \mathrm{F}$ amplitude, and $\delta$ is the measure of force sensitivity of the myosin molecule. $k_{0}$ and $\delta$ are the parameters that vary in mutant myosins as compared to WT. These values can be obtained by fitting the spread of the $k_{d}$ values at different external forces with the equation S1. During each stroking event, the positions of the dumbbells are shifted accordingly and therefore, the step sizes for individual myosin molecules can also be obtained by analyzing the same HFS data ${ }^{13}$. The data reported in this paper are gathered from the interactions of multiple different individual myosin molecules with different actin dumbbells during independent experiments done over several different days.

\section{Actin-activated ATPase assay}

Actin-activated ATPase rates were measured using an NADH-coupled assay as previously described $^{13,55}$. Actin was prepared as described previously ${ }^{56}$ and dialyzed $4 x$ into assay buffer: 5 $\mathrm{mM} \mathrm{KCl}, 10 \mathrm{mM}$ imidazole $\mathrm{pH}$ 7.5, $3 \mathrm{mM} \mathrm{MgCl}_{2}$, and $1 \mathrm{mM}$ DTT. Actin was then mixed with a 1:50-200 molar ratio of gelsolin (prepared from E. coli as described previously ${ }^{57}$ ), mixed thoroughly, and incubated on ice for $>30$ minutes. In a clear 96 -well plate with $100 \mathrm{uL}$ final volume, actin*gelsolin was mixed with assay buffer to achieve $0-80 \mu \mathrm{M}$ final concentrations and enough myosin to achieve $25 \mathrm{nM}$ final concentration. To measure the basal ATPase rate, myosin was added without actin at final concentrations of $75-125 \mathrm{nM}$. The plate was then incubated at room temperature for 10 minutes with constant shaking. To initiate the reaction, $20 \mathrm{uL}$ of a $5 x$ coupling solution containing $100 \mathrm{U} / \mathrm{mL}$ lactate dehydrogenase (product \#L1254, Sigma-Aldrich, St. Louis, MO, USA), $500 \mathrm{U} / \mathrm{mL}$ pyruvate kinase (product \#500-20, Lee Biosolutions, Maryland Heights, MO, USA), $2.5 \mathrm{mM}$ phospho(enol) pyruvate (Sigma P0564), $10 \mathrm{mM}$ ATP, and $2 \mathrm{mM}$ NADH (Sigma 
N8129). The plate was again incubated for 2 - 5 minutes at room temperature with shaking before reading absorbance at $340 \mathrm{~nm}$ every 15 - 30 seconds for 15 - 25 minutes. A standard curve of ADP from 0 - $300 \mu \mathrm{M}$ was created to convert the absorbance values to concentration of ADP produced.

Rates for each concentration of actin were calculated from the slope of a plot of [ADP] produced over time. For each concentration of actin, technical triplicates were performed on the same plate. These rates were divided by the concentration of myosin in each well, plotted against the concentration of actin, and fitted to Michaelis-Menten kinetics to obtain the values in columns 4 and 5 of table S1, where the error reported is the standard error of the fit. Each mutant 2- and 25hep was prepared in tandem with a paired WT 2-hep control and measured with technical triplicates (on a single plate) of two independent biological replicates (different days, freshly prepared each day). To obtain plots in figures 2 and 5, rates from one biological replicate were normalized to the $k_{\text {cat }}$ 's of the same-day WT 2-hep and mutant 2-hep, respectively. A t-test was used to compare the WT 2-hep to the mutant 2-hep rates.

\section{Single ATP turnover assays}

To determine data reported in Fig. 6, Fig. S2, and table S2, a single mant-ATP turnover assay was used as described previously ${ }^{24}$. Briefly, myosin was buffer exchanged $5 \mathrm{X}$ into assay buffer containing $100 \mathrm{mM} \mathrm{KOAc,} 10 \mathrm{mM}$ Tris $\mathrm{pH}$ 7.5, $1 \mathrm{mM}$ DTT, $4 \mathrm{mM} \mathrm{MgCl}_{2}$, and $1 \mathrm{mM}$ EDTA in a 50 or $100 \mathrm{kDa}$ cutoff $0.5 \mu \mathrm{L}$ Amicon filter. Myosin was then mixed with appropriate volumes of assay buffer containing $0 \mathrm{mM} \mathrm{KOAc}$ and $100 \mathrm{mM} \mathrm{KOAc}$ to achieve final salt concentrations of $5 \mathrm{mM}$ (for 25-hep myosin) or 25 mM (for 2-hep myosin-2-hep does not show a dependence on salt concentration in this assay ${ }^{24}$ ) KOAc and myosin concentrations of $200-900 \mathrm{nM}$ with a $100 \mu \mathrm{L}$ final assay volume. This was added to a 96-well black plate, where only a single well was measured at a time. 2'-(or-3')-O-(N-Methylanthraniloyl) adenosine 5'-triphosphate (mant-ATP, Thermo-Fisher Scientific, Waltham, MA, USA) was serially diluted to concentrations of $5-15 \mu \mathrm{M}$ in assay buffer and added to the myosin at a final concentration of $1 \mathrm{X}-1.2 \mathrm{X}$ the final concentration of myosin. Within 10 - 20 seconds, excess unlabeled ATP ( $4 \mathrm{mM}$ final concentration) was added to the myosin + mant-ATP mixture, and the fluorescent signal (470 nm Em/405 nm Ex) was measured every $\sim 2 \mathrm{~s}$ for $16 \mathrm{~min}$. The "dead time" between adding unlabeled ATP and the first fluorescence measurement was recorded for each well and added to the time measured by the plate reader. Fluorescence signal vs. time was plotted for each replicate and fitted to a fiveparameter bi-exponential decay. Ambiguous fits were discarded. Average fast rates, slow rates, 
percent fast phase decay, and percent slow phase decay (with their SEMs) are presented in table S2. For Fig. 6A-F, representative curves for each protein were normalized to the fitted $Y_{0}=1.0$ and plateau value $=0.0$ and fitted again to a five-parameter bi-exponential decay. They are plotted alongside simulated single exponential curves that have the fast rates and slow rates, respectively.

\section{Light chain loading gel assay}

Loading of the ELC and RLC was determined using denaturing SDS-PAGE. Before analysis, WT and mutant 2-hep myosins were buffer exchanged $5 \mathrm{X}$ in a 50 or $100 \mathrm{kDa} 0.5 \mu \mathrm{L}$ Amicon filter to remove any light chains that were unbound to the heavy chain. Myosin samples were loaded in a dilution series across the gel at 10 pmol, 5 pmol, 3 pmol, 2 pmol, and 1 pmol per lane. After separation by gel electrophoresis, the gel was stained in Coomassie, destained, and its fluorescence was scanned at $700 \mathrm{~nm}$ with a LI-COR Odyssey imaging system. Each band was quantified using $\mathrm{Fiji}^{58}$. A plot of raw integrated density versus pmol protein loaded was generated for each light chain and the heavy chain of each protein sample. Non-linear points were removed (due to the much higher molecular weight of the heavy chain as compared to the light chains, the linear range does not fully overlap--for example, the 10 pmol load is generally non-linear with the $5,3,2$, and 1 pmol loads for the heavy chain) and a linear fit was generated for each light chain and the heavy chain of each protein sample separately. The slope of each light chain fit was divided by the slope of the heavy chain fit for each protein sample, respectively, to give raw data as presented for WT 2-hep in Fig. S1B. Expected ratios based on the molecular weights of the light chains and heavy chain are noted in the legend of Fig. S1, but Coomassie staining is biased by amino acid identity in each protein sample, thus it not unusual that measured ratios deviate somewhat from expected values based on molecular weight alone. Fig. S3 shows light chain ratios for each mutant 2-hep normalized to their same-day WT 2-hep controls. P-values were determined using a paired t-test, where each mutant 2-hep was paired with its same-day WT 2hep control. 
Acknowledgements: We wish to acknowledge Chao Liu for training, support, and commentary, especially with regards to the optical trapping experiments. We also thank Masataka Kawana, Neha Nandwani, Darshan Trivedi, Divya Pathak, and Alison Schroer Vander Roest in the Spudich group for useful comments. We also want to thank our collaborators Leslie Leinwand and Thomas Perkins and their research group members, who provided comments and support throughout the project.

Funding: This work was funded by NIH grants HL117138 and 2GM033289 to JAS and KMR. MMM was supported by Stanford Cellular and Molecular Biology Training Grant T32GM007276.

Author contributions: MMM, KMR, and JAS conceived the project and experiments. MMM expressed and purified all proteins and performed and analyzed actin-activated ATPase experiments, single turnover experiments, light chain-loading experiments, and in vitro motility experiments. DB carried out and analyzed optical trapping experiments. AD and KMR prepared adenovirus for protein expression. KMR cloned myosin constructs for adenoviral expression. MMM prepared the written manuscript with support and commentary from DB, KMR, and JAS.

Competing interests: JAS is cofounder and on the Scientific Advisory Board of Cytokinetics, Inc., a company developing small molecule therapeutics for treatment of hypertrophic cardiomyopathy. 


\section{REFERENCES}

1. Rayment, I. et al. Structure of the actin-myosin complex and its implications for muscle contraction. Science 261, 58-65 (1993).

2. Rayment, I. et al. Three-dimensional structure of myosin subfragment-1: a molecular motor. Science 261, 50-58 (1993).

3. Uyeda, T. Q., Abramson, P. D. \& Spudich, J. A. The neck region of the myosin motor domain acts as a lever arm to generate movement. Proc. Natl. Acad. Sci. U. S. A. 93, 4459-4464 (1996).

4. Geeves, M. A. \& Holmes, K. C. Structural mechanism of muscle contraction. Annu. Rev. Biochem. 68, 687-728 (1999).

5. Spudich, J. A. \& Sivaramakrishnan, S. Myosin VI: an innovative motor that challenged the swinging lever arm hypothesis. Nat. Rev. Mol. Cell Biol. 11, 128-137 (2010).

6. Ho, C. Y. et al. Echocardiographic Strain Imaging to Assess Early and Late Consequences of Sarcomere Mutations in Hypertrophic Cardiomyopathy. Circ. Cardiovasc. Genet. 2, 314-321 (2009).

7. Nag, S. et al. Contractility parameters of human $\beta$-cardiac myosin with the hypertrophic cardiomyopathy mutation R403Q show loss of motor function. Sci. Adv. 1, e1500511 (2015).

8. Adhikari, A. S. et al. Early-Onset Hypertrophic Cardiomyopathy Mutations Significantly Increase the Velocity, Force, and Actin-Activated ATPase Activity of Human $\beta$-Cardiac Myosin. Cell Rep. 17, 2857-2864 (2016).

9. Kawana, M., Sarkar, S. S., Sutton, S., Ruppel, K. M. \& Spudich, J. A. Biophysical properties of human $\beta$-cardiac myosin with converter mutations that cause hypertrophic cardiomyopathy. Sci. Adv. 3, 1601959 (2017).

10. Nag, S. et al. The myosin mesa and the basis of hypercontractility caused by hypertrophic cardiomyopathy mutations. Nat. Struct. Mol. Biol. 24, 525 (2017).

11. Adhikari, A. S. et al. $\beta$-Cardiac myosin hypertrophic cardiomyopathy mutations release sequestered heads and increase enzymatic activity. Nat. Commun. 2019101 10, 1-10 (2019).

12. Sarkar, S. S. et al. The hypertrophic cardiomyopathy mutations $R 403 Q$ and $R 663 H$ increase the number of myosin heads available to interact with actin. Sci. Adv. 6, 69 (2020).

13. Vander Roest, A. S. et al. Hypertrophic cardiomyopathy $\beta$-cardiac myosin mutation 
(P710R) leads to hypercontractility by disrupting super relaxed state. Proc. Natl. Acad. Sci. U. S. A. 118, (2021).

14. Spudich, J. A. Three perspectives on the molecular basis of hypercontractility caused by hypertrophic cardiomyopathy mutations. Pflugers Arch. 471, 701-717 (2019).

15. Liu, C., Kawana, M., Song, D., Ruppel, K. M. \& Spudich, J. A. Controlling load-dependent kinetics of $\beta$-cardiac myosin at the single-molecule level. Nat. Struct. Mol. Biol. 25, 505514 (2018).

16. Semsarian, C., Ingles, J., Maron, M. S. \& Maron, B. J. New Perspectives on the Prevalence of Hypertrophic Cardiomyopathy. J. Am. Coll. Cardiol. 65, 1249-1254 (2015).

17. Konno, T., Chang, S., Seidman, J. G. \& Seidman, C. E. Genetics of hypertrophic cardiomyopathy. Curr. Opin. Cardiol. 25, 205-209 (2010).

18. Tyska, M. J. et al. Single-Molecule Mechanics of R403Q Cardiac Myosin Isolated From the Mouse Model of Familial Hypertrophic Cardiomyopathy. Circ. Res. 86, 737-744 (2000).

19. Debold, E. et al. Hypertrophic and dilated cardiomyopathy mutations differentially affect the molecular force generation of mouse alpha-cardiac myosin in the laser trap assay. Am. J. Physiol. Heart Circ. Physiol. 293, (2007).

20. Moore, J. R., Leinwand, L. \& Warshaw, D. M. Understanding Cardiomyopathy Phenotypes Based on the Functional Impact of Mutations in the Myosin Motor. Circ. Res. 111, 375-385 (2012).

21. Spudich, J. A. The myosin mesa and a possible unifying hypothesis for the molecular basis of human hypertrophic cardiomyopathy. Biochem. Soc. Trans. 43, 64 (2015).

22. Alamo, L. et al. Effects of myosin variants on interacting-heads motif explain distinct hypertrophic and dilated cardiomyopathy phenotypes. Elife 6, (2017).

23. Robert-Paganin, J., Auguin, D. \& Houdusse, A. Hypertrophic cardiomyopathy disease results from disparate impairments of cardiac myosin function and auto-inhibition. Nat. Commun. 2018919 , 1-13 (2018).

24. Anderson, R. L. et al. Deciphering the super relaxed state of human $\beta$-cardiac myosin and the mode of action of mavacamten from myosin molecules to muscle fibers. Proc. Natl. Acad. Sci. U. S. A. 115, E8143-E8152 (2018).

25. Trybus, K. M. Assembly of cytoplasmic and smooth muscle myosins. Curr. Opin. Cell Biol. 3, 105-111 (1991).

26. Wendt, T., Taylor, D., Trybus, K. M. \& Taylor, K. Three-dimensional image reconstruction of dephosphorylated smooth muscle heavy meromyosin reveals asymmetry in the 
interaction between myosin heads and placement of subfragment 2. Proc. Natl. Acad. Sci. 98, 4361-4366 (2001).

27. Woodhead, J. L. et al. Atomic model of a myosin filament in the relaxed state. Nature 436, 1195-1199 (2005).

28. Jung, $\mathrm{H}$. et al. Conservation of the regulated structure of folded myosin 2 in species separated by at least 600 million years of independent evolution. Proc. Natl. Acad. Sci. U. S. A. 105, 6022-6026 (2008).

29. AL-Khayat, H. A., Kensler, R. W., Squire, J. M., Marston, S. B. \& Morris, E. P. Atomic model of the human cardiac muscle myosin filament. Proc. Natl. Acad. Sci. U. S. A. 110, 318 (2013).

30. Lee, K. H. et al. Interacting-heads motif has been conserved as a mechanism of myosin II inhibition since before the origin of animals. Proc. Natl. Acad. Sci. U. S. A. 115, E1991 (2018).

31. Stewart, M. A., Franks-Skiba, K., Chen, S. \& Cooke, R. Myosin ATP turnover rate is a mechanism involved in thermogenesis in resting skeletal muscle fibers. Proc. Natl. Acad. Sci. 107, 430-435 (2010).

32. McNamara, J. W., Li, A., dos Remedios, C. G. \& Cooke, R. The role of super-relaxed myosin in skeletal and cardiac muscle. Biophys. Rev. 2014 71 7, 5-14 (2014).

33. Rohde, J. A., Roopnarine, O., Thomas, D. D. \& Muretta, J. M. Mavacamten stabilizes an autoinhibited state of two-headed cardiac myosin. Proc. Natl. Acad. Sci. U. S. A. 115, E7486 (2018).

34. Trybus, K. M., Freyzon, Y., Faust, L. \& Sweeney, H. Spare the rod, spoil the regulation: necessity for a myosin rod. Proc. Natl. Acad. Sci. U. S. A. 94, 48-52 (1997).

35. Chu, S., Muretta, J. M. \& Thomas, D. D. Direct detection of the myosin super-relaxed state and interacting-heads motif in solution. J. Biol. Chem. 101157 (2021). doi:10.1016/J.JBC.2021.101157

36. Nag, S. \& Trivedi, D. V. To lie or not to lie: Super-relaxing with myosins. Elife 10, 1-21 (2021).

37. Houdusse, A. M., Szent-Gyorgyi, A. G. \& Cohen, C. Three conformational states of scallop myosin S1. Proc. Natl. Acad. Sci. U. S. A. 97, 11238-11243 (2000).

38. Houdusse, A. M. \& Cohen, C. Structure of the regulatory domain of scallop myosin at $2 \mathrm{~A}$ resolution: implications for regulation. Structure 4, 21-32 (1996).

39. Himmel, D. M., Mui, S., O’Neall-Hennessey, E., Szent-Györgyi, A. G. \& Cohen, C. The on-off switch in regulated myosins: different triggers but related mechanisms. J. Mol. Biol. 
394, 496-505 (2009).

40. Pylypenko, O. \& Houdusse, A. M. Essential 'ankle' in the myosin lever arm. Proc. Natl. Acad. Sci. U. S. A. 108, 5-6 (2011).

41. Brown, J. H. et al. Visualizing key hinges and a potential major source of compliance in the lever arm of myosin. Proc. Natl. Acad. Sci. U. S. A. 108, 114-119 (2011).

42. Scarff, C. et al. Structure of the shutdown state of myosin-2. Nature 588, 515-520 (2020).

43. Yang, S. et al. Cryo-EM structure of the inhibited (10S) form of myosin II. Nat. 2020 5887838 588, 521-525 (2020).

44. Greenberg, M. J., Kazmierczak, K., Szczesna-Cordary, D. \& Moore, J. R. Cardiomyopathy-linked myosin regulatory light chain mutations disrupt myosin straindependent biochemistry. Proc. Natl. Acad. Sci. U. S. A. 107, 17403-17408 (2010).

45. Sherwood, J. J., Waller, G. S., Warshaw, D. M. \& Lowey, S. A point mutation in the regulatory light chain reduces the step size of skeletal muscle myosin. PNAS 101, (2004).

46. Karabina, A., Kazmierczak, K., Szczesna-Cordary, D. \& Moore, J. R. Myosin regulatory light chain phosphorylation enhances cardiac $\beta$-myosin in vitro motility under load. Arch. Biochem. Biophys. 580, 14 (2015).

47. Farman, G. P., Muthu, P., Kazmierczak, K., Szczesna-Cordary, D. \& Moore, J. R. Impact of familial hypertrophic cardiomyopathy-linked mutations in the $\mathrm{NH} 2$ terminus of the RLC on $\beta$-myosin cross-bridge mechanics. https://doi.org/10.1152/japp/physiol.00798.2014 117, 1471-1477 (2014).

48. Homburger, J. R. et al. Multidimensional structure-function relationships in human $\beta$ cardiac myosin from population-scale genetic variation. Proc. Natl. Acad. Sci. U. S. A. 113, 6701-6 (2016).

49. Aksel, T., Yu, E. C., Sutton, S., Ruppel, K. M. \& Spudich, J. A. Ensemble Force Changes that Result from Human Cardiac Myosin Mutations and a Small-Molecule Effector. Cell Rep. 11, 910-920 (2015).

50. Sung, J. et al. Harmonic force spectroscopy measures load-dependent kinetics of individual human $\beta$-cardiac myosin molecules. Nat. Commun. 6, (2015).

51. Sung, J., Mortensen, K. I., Spudich, J. A. \& Flyvbjerg, H. How to Measure LoadDependent Kinetics of Individual Motor Molecules Without a Force-Clamp. Methods Enzymol. 582, 1 (2017).

52. Greenberg, M. J., Shuman, H. \& Ostap, E. M. Inherent Force-Dependent Properties of $\beta$ Cardiac Myosin Contribute to the Force-Velocity Relationship of Cardiac Muscle.

Biophys. J. 107, L41-L44 (2014). 
53. Gourinath, S. et al. Crystal Structure of Scallop Myosin S1 in the Pre-Power Stroke State to $2.6 \AA ̊$ Resolution: Flexibility and Function in the Head. Structure 11, 1621-1627 (2003).

54. Sommese, R. F. et al. Molecular consequences of the R453C hypertrophic cardiomyopathy mutation on human $\beta$-cardiac myosin motor function. Proc. Natl. Acad. Sci. 110, 12607-12612 (2013).

55. De La Cruz, E. M. \& Ostap, E. M. Kinetic and equilibrium analysis of the myosin ATPase. Methods Enzymol. 455, 157-192 (2009).

56. Spudich, J. A. \& Watt, S. The regulation of rabbit skeletal muscle contraction. I.

Biochemical studies of the interaction of the tropomyosin-troponin complex with actin and the proteolytic fragments of myosin. J. Biol. Chem. 246, 4866-4871 (1971).

57. Dawson, J. F., Sablin, E. P., Spudich, J. A. \& Fletterick, R. J. Structure of an F-actin trimer disrupted by gelsolin and implications for the mechanism of severing. J. Biol. Chem. 278, 1229-1238 (2003).

58. Schindelin, J. et al. Fiji: an open-source platform for biological-image analysis. Nat. Methods 9, 676-682 (2012). 